Published in final edited form as:

Nat Chem Biol. 2018 April ; 14(4): 352-360. doi:10.1038/s41589-018-0004-9.

\title{
A robotic multidimensional directed evolution approach applied to fluorescent voltage reporters
}

\author{
Kiryl D. Piatkevich ${ }^{1,+}$, Erica E. Jung ${ }^{1,+}$, Christoph Straub ${ }^{2}$, Changyang Linghu ${ }^{1,3}$, Demian \\ Park $^{1}$, Ho-Jun Suk ${ }^{1,4}$, Daniel R. Hochbaum ${ }^{2}$, Daniel Goodwin ${ }^{1}$, Eftychios Pnevmatikakis ${ }^{5}$, \\ Nikita Pak ${ }^{1,6}$, Takashi Kawashima ${ }^{7}$, Chao-Tsung Yang ${ }^{7}$, Jeffrey L. Rhoades ${ }^{8}$, Or Shemesh ${ }^{1}$, \\ Shoh Asano ${ }^{1}$, Young-Gyu Yoon ${ }^{1,3}$, Limor Freifeld ${ }^{1}$, Jessica L. Saulnier ${ }^{2}$, Clemens \\ Riegler $^{9,10}$, Florian Engert ${ }^{9}$, Thom Hughes ${ }^{11}$, Mikhail Drobizhev ${ }^{11}$, Balint Szabo ${ }^{12}$, Misha B. \\ Ahrens $^{7}$, Steven W. Flavell ${ }^{8}$, Bernardo L. Sabatini ${ }^{2}$, and Edward S. Boyden 1,13,14,15,16, ${ }^{*}$ \\ ${ }^{1}$ Media Lab, Massachusetts Institute of Technology (MIT), Cambridge, Massachusetts, USA \\ ${ }^{2}$ Howard Hughes Medical Institute, Department of Neurobiology, Harvard Medical School, Boston, \\ Massachusetts, USA \\ ${ }^{3}$ Department of Electrical Engineering and Computer Science, MIT, Cambridge, Massachusetts, \\ USA
}

${ }^{4}$ Harvard-MIT Division of Health Sciences and Technology, MIT, Cambridge, Massachusetts, USA ${ }^{5}$ Simons Center Data Analysis, Simons Foundation, New York, New York, USA

${ }^{6}$ Department of Mechanical Engineering, MIT, Cambridge, Massachusetts, USA

${ }^{7}$ Janelia Research Campus, Howard Hughes Medical Institute, Ashburn, Virginia, USA

${ }^{8}$ Picower Institute for Learning \& Memory and Department of Brain \& Cognitive Sciences, MIT, Cambridge, Massachusetts, USA

${ }^{9}$ Department of Molecular and Cellular Biology and Center for Brain Science, Harvard University, Cambridge, Massachusetts, USA

${ }^{10}$ Department of Neurobiology, Faculty of Life Sciences, University of Vienna, Wien, Austria

\footnotetext{
Users may view, print, copy, and download text and data-mine the content in such documents, for the purposes of academic research, subject always to the full Conditions of use: http://www.nature.com/authors/editorial_policies/license.html\#terms

*Correspondence to esb@media.mit.edu.

+These authors contributed equally.

AUTHOR CONTRIBUTIONS

K.D.P., E.E.J., and E.S.B. initiated the project, made high-level designs and plans, and interpreted data. K.D.P., E.E.J., B.S. and O.S. developed the hierarchical multiparameter screening approach. K.D.P. and E.E.J. developed miRFP and together with C.L., M.D., T.H., H.S., and S.A. performed its characterization. K.D.P. and E.E.J. developed Archons and together with D.P. characterized them in cultured cells. C.S., D.H., J.S., and B.L.S. performed electrophysiology experiments in acute brain slices. K.D.P, E.E.J, D.G., E.P. and C.L. analyzed neuronal culture data. N. P. and Y.Y assisted on imaging setups. E.E.J. and K.D.P with help from L.F. performed experiments on zebrafish injected by C.Y., T.K., and M.B.A. K.D.P., S.W.F. and J.L.R performed experiments on C. elegans. C.R. and F.E. designed vectors for zebrafish expression. C.L. and E.E.J. performed statistical analysis. K.D.P., E.E.J., C.S., C.L., and E.S.B. wrote the paper with contributions from all authors. E.S.B. oversaw all aspects of the project.
}

COMPETING FINANCIAL INTERESTS

B.S. is a founder of the CellSorter startup company. K.D.P., E.E.J., and E.S.B. are inventors on patent applications regarding the molecules here reported. B.S., K.D.P., E.E.J., and E.S.B. are inventors on a patent application regarding the screening method here developed. 
${ }^{11}$ Department of Cell Biology and Neuroscience, Montana State University, Bozeman, Montana, USA

12Department of Biological Physics, Eotvos University, Budapest, Hungary

${ }^{13}$ Department of Biological Engineering, MIT, Cambridge, Massachusetts, USA

${ }^{14}$ MIT Center for Neurobiological Engineering, MIT, Cambridge, Massachusetts, USA

${ }^{15}$ Department of Brain and Cognitive Sciences, MIT, Cambridge, Massachusetts, USA

${ }^{16}$ MIT McGovern Institute for Brain Research, MIT, Cambridge, Massachusetts, USA

\section{Abstract}

We here present a new way to engineer complex proteins toward multidimensional specifications, through a simple yet scalable directed evolution strategy. By robotically picking mammalian cells that are identified, under a microscope, to express proteins that simultaneously exhibit several specific properties, we can screen through hundreds of thousands of proteins in a library in a matter of a few hours, evaluating each along multiple performance axes. We demonstrate the power of this approach by identifying a novel genetically encoded fluorescent voltage indicator, simultaneously optimizing brightness and membrane localization of the protein using our microscopy-guided cell picking strategy. We produced the high-performance opsin-based fluorescent voltage reporter Archon1, and demonstrated its utility by imaging spiking and millivolt-scale subthreshold and synaptic activity in acute mouse brain slices as well as in larval zebrafish in vivo. We also demonstrate measurement of postsynaptic responses downstream of optogenetically controlled neurons in C. elegans.

\section{Keywords}

Protein engineering; robotics; directed molecular evolution; voltage imaging; mouse; C. elegans; optogenetics; zebrafish; synaptic transmission; subthreshold activity

\section{INTRODUCTION}

Directed molecular evolution is a powerful strategy for protein engineering ${ }^{1,2}$. Most strategies for screening libraries of mutated genes for improved phenotypes either exhibit high throughput but are limited to the detection of fluorescence ${ }^{2}$, or can extract multiple protein functional parameters but exhibit modest throughput or complexity of usage that challenges end user protein engineers ${ }^{3-7}$. In order to enable the simple and high throughput directed evolution of proteins so that multiple properties can each be simultaneously optimized toward specific goals, we developed a strategy for robotically picking mammalian cells that are identified, under a microscope, to express proteins with appropriate properties. Our strategy is inexpensive and easily used by protein engineers, and enables the assessment of hundreds of thousands of protein variants in a few hours.

We applied our strategy to the creation of genetically encoded fluorescent indicators of membrane potential ${ }^{8}$. An ideal genetically encoded fluorescent voltage indicator would localize well to the plasma membrane, be bright and exhibit high signal-to-noise ratio 
(SNR), exhibit large and linear fluorescent changes in response to voltage fluctuations, respond to changes in voltage rapidly enough to preserve the fidelity of spiking, exhibit stable (i.e., non-photobleaching) fluorescence over timescales appropriate for conducting a biological experiment, and be compatible with optogenetic control of neural activity ${ }^{9}$. To date it has remained difficult to simultaneously optimize all of these properties - perhaps because directed evolution methods that select for one property may de-optimize other properties ${ }^{10}$, and also the optimization may need to be done in mammalian cells to ensure high performance in neurons ${ }^{3,11}$.

There are two classes of genetically encoded voltage reporters ${ }^{8}-$ one uses the fluorescence of microbial opsins (e.g., archaerhodopsin-3 (Arch)-based fluorescent voltage reporters ${ }^{12,13}$ ) to report neural activity, and the other couples a GFP-like fluorescent protein to a voltage sensing membrane protein (e.g., a voltage-sensitive phosphatase ${ }^{14}$ or an opsin $\left.{ }^{15,16}\right)$. Fluorescent opsins have been relatively dim, and suffer from poor localization, and thus exhibit low SNR (Supplementary Tables 1,2). GFP-like fluorescent protein-containing reporters are brighter, but have to date exhibited small fractional changes in fluorescence, photobleach rapidly, and are incompatible with optogenetic control due to spectral overlap (Supplementary Tables 1,2). We used robotic cell picking to screen mammalian cells expressing individual members of a library of fluorescent voltage sensor candidates, based upon brightness, localization, and photostability. The result, the fluorescent voltage sensor Archon1 (so named because it is based on Arch, with 13 point mutations), exhibits excellent localization, SNR, sensitivity, response speed, photostability, and full compatibility with optogenetic control. It is also severalfold brighter than previous opsin-based fluorescent voltage reporters that are red light sensitive. We demonstrate the utility of Archon1 in multiple species by imaging subthreshold (e.g., $\sim 5 \mathrm{mV}$ ) synaptic responses in mouse cortical brain slices, high speed spiking and subthreshold activity in larval zebrafish brain, and neural responses downstream of optogenetically controlled neurons in C. elegans.

\section{RESULTS}

\section{Robotic multidimensional directed evolution of proteins}

In order to be able to screen large numbers of mutant genes along multiple parameters, we combined microscopy and image analysis with robotic cell picking (Fig. 1a). We adapted a computer-vision-guided automated micropipette capable of controlled suction and positive pressure, and thus the isolation of single cells (see Methods) ${ }^{17,18}$, so that we could screen 300,000 cells expressing different constructs in $\sim 4$ hours. We transfected a gene library into HEK293T cells so that transfected cells would receive from one to four plasmids per cell (details of characterization in Supplementary Fig. 1), eliminated dead and nontransfected cells using FACS, and then performed multiple rounds of microscopy-guided cell picking to examine multiple parameters (e.g., brightness, membrane localization; Supplementary Fig. 2).

To validate the overall workflow, we performed three rounds of directed molecular evolution to develop a monomeric near-infrared fluorescent protein (FP) from the $R p \mathrm{BphP} 1$ bacteriophytochrome ${ }^{19}$ (see Supplementary Table 3 for screening parameters; screening progress is described in Supplementary Fig. 3). The final selected variant, named miRFP, 
had absorbance and emission maxima at $674 \mathrm{~nm}$ and $703 \mathrm{~nm}$, respectively, and demonstrated monomeric state both in vitro and in cultured mammalian cells (Supplementary Figs. 4-6). Furthermore, miRFP exhibited higher molecular brightness than previously developed, spectrally similar near-infrared FPs (Supplementary Table 4) and could be readily expressed in neurons in culture and in vivo and imaged using both one- and two-photon microscopy (Supplementary Figs. 7).

\section{Multidimensional screening of genetically encoded voltage indicators}

We next turned to multidimensional screening for a high-performance fluorescent voltage sensor. To obtain a molecule compatible with optogenetic control, we began with a template with red fluorescence (since optogenetic controllers are sensitive to blue light, ideally we would have a voltage reporter that would be illuminated by orange or red light). We began with the opsin core of the previously reported voltage sensor QuasAr2, with a fluorescence excitation maxima at $590 \mathrm{~nm}^{12}$. For the first round of directed molecular evolution, we generated a gene library with error-prone PCR and cloned it into the expression vector. After expression of the library in HEK cells for 48 hours, we used FACS to remove nontransfected cells and cells expressing non-fluorescent (and thus non-functional) mutants, which was $>99.9 \%$ of the entire population (Supplementary Fig. 8). We then performed microscopy-guided cell picking to screen for cells containing genes whose products exhibited exemplary brightness and membrane localization, simultaneously (see Supplementary Table 3 for screen imaging parameters). We also assessed, in a subset of these cells, fluorescence photostability by taking time-lapse images under continuous illumination, but found that the variants selected already had great photostability, and as measuring photostability is time-consuming, we halted this specific part of the analysis. Selected cells were those exhibiting high-performing combinations of membrane localization and fluorescence brightness along the Pareto frontier ${ }^{20}$ (i.e., the set of cells that dominate other cells in at least one parameter), as previously used to computationally evaluate progress in the context of multiobjective evolutionary optimisation ${ }^{21-23}$ (Fig. 1b; see Methods for details of the step-by-step Pareto frontier algorithm). These genes were then rescreened for brightness, membrane localization, and voltage sensitivity (see Methods), yielding a quantitative three-parameter metric. We calculated the product of these three measured parameters (each normalized to its maximum value, to ensure the roughly equal contribution of each parameter to the product), and then the five variants with the highest such products were selected for sequencing. Sequence analysis revealed four amino acid positions, namely T56, T117, T183 and L198, that were changed in four out of the five variants, and we also identified four amino acid mutations in a-helices (T20S, L31V, K47R, and A137T) and two mutations in $\beta$-strands (S80P and D88N) which were represented at least once in the selected mutants (Supplementary Fig. 9).

For the second round of directed molecular evolution, we generated a site-directed library of variants containing mutations identified in our first round, as well as mutations near the Schiff base linkage (some of which had been previously explored ${ }^{13,24}$ ). We repeated the screen as in the first round, including the assessment of a subset of the clones for photostability. Since the method of electrical stimulation used in the screen was not very quantitative ${ }^{25,26}$, we characterized the top 21 variants via whole-cell patch clamp and 
concurrent imaging. Discarding mutants with kinetics (i.e., $\boldsymbol{\tau}_{\mathrm{on}}$ or $\boldsymbol{\tau}_{\mathrm{off}}$ ) slower than $10 \mathrm{~ms}$, and using the product of normalized brightness, localization and voltage sensitivity as the final ranking criterion, we obtained seven final candidates with improved brightness and membrane localization, of which two exhibited higher voltage sensitivity (Supplementary Figs. 10,11). Prioritizing localization as the key parameter, with brightness and voltage sensitivity as secondary parameters, we chose two molecules - denoted Archon1 and Archon 2 - for further investigation. Archon1 and Archon2 localized well on the plasma membrane of HEK cells (Fig. 1c,d), and exhibited 2.4- and 6.8-fold increased brightness, respectively, over the parental template (Fig. 1e). Fluorescence changes $(\Delta \mathrm{F} / \mathrm{F})$ in HEK cells for Archon 1 and Archon 2 over $100 \mathrm{mV}$ voltage swings were $81 \pm 8 \%$ and $20 \pm 2 \%$, respectively, compared to $46 \pm 4 \%$ for the template (mean \pm standard deviation throughout; Fig. 1f,g). We investigated the contribution of specific point mutations to changes in localization, brightness, voltage sensitivity, and kinetics, and found the patterns that emerged to be complex (Supplementary Table 6), with a given mutation often improving one parameter but worsening another. Thus, simultaneous multidimensional hierarchical screening may be key for generating practical fluorescent voltage indicators that excel along multiple axes at once.

\section{Biophysical properties of Archons}

We fused Archons with EGFP for visualization, and Golgi export trafficking signal (KGC) and endoplasmic reticulum export (ER) sequences ${ }^{27,28}$ (Fig. 2a) which are widely used to help archaeal opsins express better in vivo (although Archon1 had excellent membrane localization in cultured neurons without them; Supplementary Fig. 12). Archons demonstrated excellent membrane localization in cultured mouse neurons (see Supplementary Fig. 13 for images of neurons expressing other sensors, under the same promoter and imaged after the same time period). Focusing on the Arch-derived voltage sensors (QuasAr2-mOrange and Archer1-EGFP, abbreviated as QuasAr2 and Archer1 in Fig. 2), we found greater brightness for Archons over earlier molecules (Fig. 2b) in cultured neurons. Archon expression did not alter membrane resistance, membrane capacitance, or resting potential of cultured neurons (Supplementary Fig. 14).

When we expressed Archon2 in mouse brain slices, we obtained lower SNR and membrane localization than with Archon1 (see below); therefore we focus on Archon1 in the main text (but we include the Archon2 data in supplementary figures). In cultured neurons, we found that Archon1 exhibited a $\Delta \mathrm{F} / \mathrm{F}$ of $43 \pm 5 \%$ (Fig. 2c-e) for a $100 \mathrm{mV}$ deflection, with a linear voltage dependence (Fig. 2e; compare to other indicators described in Supplementary Table 1). The speed of response of Archon 1 was fast, with a biexponential response to a $100 \mathrm{mV}$ voltage step with time constants of onset of $0.61 \pm 0.06 \mathrm{~ms}$ ( $88 \%$ of total amplitude) and 8.1 $\pm 0.5 \mathrm{~ms}$ (remaining amplitude), and a time constant of inactivation of $1.1 \pm 0.2 \mathrm{~ms}$ ( $88 \%$ of total amplitude) and $13 \pm 3 \mathrm{~ms}$ (remaining amplitude) (Fig. 2c). Archon1 fluorescence was able to follow small, high-speed changes in voltage in cultured neurons, including fewmillivolt voltage transients, as well as action potentials, with the latter broadening by a few hundred microseconds in waveform duration, and for which Archon 1 exhibited a $\Delta \mathrm{F} / \mathrm{F}$ of 30 $\pm 6 \%$ and SNR of 36 (Fig. 2f-h, Supplementary Fig. 15). Archon2 exhibited faster kinetics but lower voltage sensitivity than Archon1 (Supplementary Fig. 16 and Supplementary Table 
1). Both Archons demonstrated linear dependence of fluorescence intensity vs. $637 \mathrm{~nm}$ excitation light power, which suggests that fluorescence was a single-photon process (Supplementary Fig. 16h).

Photobleaching limits the impact of voltage imaging in neuroscience, since signal decreases result in signal eventually blending in with noise. We excited Archons with $800 \mathrm{~mW} / \mathrm{mm}^{2}$ $637 \mathrm{~nm}$ light for 900 seconds, and found that Archon1 retained $95 \pm 16 \%$ of its baseline fluorescence (Fig. 2i), far better retention of fluorescence than Archon2, QuasAr2, Archer1 and Ace (Supplementary Table 1). Thus, Archon1 may be able to support voltage imaging over timescales relevant to behavior.

As Archons are derived from Arch, a light-driven proton pump, we characterized their responses to illumination with $470 / 20 \mathrm{~nm}$ light at $15 \mathrm{~mW} / \mathrm{mm}^{2}$ (as used to image EGFP) and $637 \mathrm{~nm}$ at $800 \mathrm{~mW} / \mathrm{mm}^{2}$ (as used for voltage imaging). Under all tested wavelengths, Archons showed no steady state photocurrent (Supplementary Fig 17). Under repetitive pulses of blue illumination, the first pulse generated a transient photocurrent of $-8 \pm 6 \mathrm{pA}$ for a few milliseconds ( $\mathrm{n}=8$ cells from one culture), as did subsequent pulses (Supplementary Fig. 17). Under repetitive pulses of red illumination, Archon1 showed a brief ( $<5 \mathrm{~ms}$ ) transient photocurrent of $-33 \pm 25 \mathrm{pA}$, while subsequent pulses of red light produced no photocurrent (Supplementary Fig. 17). Archon2 showed no photocurrents under any condition (Supplementary Fig. 17). Buoyed by these results, we measured changes in the red fluorescence of Archons under blue light intensities typically used for optogenetic control $^{4}$; in particular, under blue light at $4.8 \mathrm{~mW} / \mathrm{mm}^{2}$ and with red illumination as above, Archons showed $<2 \%$ changes in fluorescence (Supplementary Figs. 15, 16) This property allowed us to use Archon1 in conjunction with the channelrhodopsin $\mathrm{CoChR}^{4,29}$ for alloptical interrogation of neurons, driving and monitoring action potentials with light (Supplementary Fig. 18). Using Archons, one could image voltage fluctuations in neuronal processes in culture, and even single dendritic spines without averaging (Supplementary Fig. 19).

\section{Use of Archons for synaptic and spiking imaging in intact mouse cortical slices}

We expressed Archons in cortical pyramidal neurons via in utero electroporation (IUE; Supplementary Fig. 20). We performed voltage-clamp recordings from L2/3 pyramidal neurons in acute brain slices from 3-4 week old mice, and simultaneously monitored Archon1 fluorescence at the cell body (Fig. 3a,b). Archon1 expressed in vivo showed good membrane localization in cell bodies and processes without aggregation, and illumination with red light did not alter membrane properties (Fig. 3b and Supplementary Figs. 20-22), whereas QuasAr2 and Archer1 showed aggregation in cell bodies and processes (Supplementary Fig. 23). We used a series of voltage-steps in voltage-clamp mode to test the ability of Archons to report membrane voltage. Stepping the holding-potential $\left(\mathrm{V}_{\mathrm{m}}\right)$ from -90 to $+10 \mathrm{mV}$ resulted in step-like fluorescent signals for Archon1 (Supplementary Fig. 24; Archon $1: \Delta \mathrm{F} / \mathrm{F}$ per $100 \mathrm{mV}: 23.5 \pm 9.3 \%$ ). The on- and off-kinetics of Archons were well described by a double-exponential function, and reached steady state within a few ms (Supplementary Fig. 24). These data suggested that Archon1 should be sensitive enough to report subthreshold voltage events and fast enough to report individual action potentials in 
acute brain slices. To test this, we injected a series of $2 \mathrm{~ms}$ current pulses with increasing amplitudes, while monitoring membrane potential and fluorescent signals. Archon1 allowed reliable detection of voltage transients from both subthreshold depolarization and action potentials (APs) under 1.5 and $15 \mathrm{~W} / \mathrm{mm}^{2}$ of excitation light (Supplementary Fig. 24). The high temporal precision and voltage sensitivity of Archon1 observed in neuron culture was borne out as excellent action potential reporting fidelity in mouse cortical neurons in brain slice (Fig. 3c-e, Supplementary Fig. 24), with excellent kinetics, dynamic range, and signal to noise when action potentials were imaged in mouse brain slices (Fig. 3f). Archon1 faithfully reported action potentials, even when elicited at the highest frequencies tested. Archon2 exhibited qualitatively similar functionality, but with reduced voltage sensitivity and SNR (Supplementary Fig. 25). Indeed, using Archon1, it was possible to image synaptic events of millivolt scale depolarization amplitude, triggered in layer $2 / 3$ with layer 5 stimulation, with excellent signal quality (Fig. $3 \mathrm{~g}$ ). Voltage deflections of $\sim 5 \mathrm{mV}$ could be observed as $\sim 2-3 \%$ fluorescence changes (Fig. 3h, left), and synaptic events this small could be imaged with signal to noise ratio of 2 or greater (Fig. 3h, right).

\section{Use of Archons for synaptic and spiking imaging in living animal brains}

We next explored the use of Archon1 in multiple in vivo contexts. Zebrafish (Danio rerio) is a species important for the study of the development and operation of the nervous system $^{30-34}$. We transiently expressed a zebrafish codon-optimized version of Archon1 (zArchon1) fused to EGFP in a subset of neurons. zArchon1, expressed in zebrafish larvae, demonstrated excellent membrane localization (Fig. 4a). zArchon1 reported action potentials with large fluorescence changes from baseline to peak (Fig. 4b,c), with excellent voltage sensitivity and SNR in larval zebrafish neurons (Fig. 4d). Notably, our voltage sensitivity and SNR are severalfold higher than those found for earlier voltage reporters in other intact neural systems (Supplementary Table 2). We assessed the photobleaching properties of zArchon1 in zebrafish larvae by applying the same illumination condition used for voltage imaging. The fluorescence of zArchon1 declined to $84 \pm 8 \%$ of baseline fluorescence over 300 seconds (Fig. 4e), or $0.05 \% / \mathrm{s}$ in zebrafish in vivo (vs. $0.01 \% / \mathrm{s}$ in cultured mouse neurons). We could observe putative subthreshold (i.e., smaller than the amplitude of spikes) events (Supplementary Fig. 26), as well as voltage recording from neuronal processes (Fig. $4 \mathrm{f}, \mathrm{g})$. As expected from the aforementioned conclusions, imaging of responses was stable over timescales of many minutes (Supplementary Fig. 27).

Finally, we explored the usage of Archon1 in the nematode C. elegans, a popular model organism in neuroscience. We used the rig-3 promoter to drive expression of codonoptimized Archon1 (wArchon1) fused to EGFP in AVA interneurons, involved in backward locomotion, while simultaneously expressing the blue light driven optogenetic controller channelrhodopsin-2 (ChR2) in an upstream neuron, the ASH neuron, under control of the sra- 6 promoter (Fig. 5a). wArchon1 demonstrated good membrane localization both at the soma and in individual axons of AVA neurons (Fig. 5b and Supplementary Fig. 28). AVA neurons, when imaged at points at the soma or along the axon, exhibited long-lasting (tens of seconds to several minutes) high and low states similar to those previously reported in AVA calcium recordings ${ }^{35}$ (Fig. 5c), with changes in fluorescence intensity relative to baseline of magnitude of $\sim 20-25 \%$ and SNR of $\sim 25-35$ (although the diversity of these 
fluctuations (Fig. 5c), in contrast to the stereotyped action potentials of vertebrate neurons, makes it difficult to arrive at a single number; $\mathrm{n}=20$ worms). The absence of blue light crosstalk with Archon function allows for combining voltage imaging using wArchon 1 with optogenetic control using opsins. When blue light pulses were delivered, 51 out of 60 blue light pulses resulted in sustained elevation of wArchon1 fluorescence in AVA neurons lasting for 38 \pm 13 seconds (Fig. $5 \mathrm{~d}, \mathrm{e} ; \Delta \mathrm{F} / \mathrm{F}$ of $\sim 16-21 \%, \sim 20-28 \mathrm{SNR}$; $\mathrm{n}=20$ worms). In contrast, when no ChR2 was present, no effect of blue light was seen (Fig. $5 \mathrm{f}$ and Supplementary Fig. 29). Finally, wArchon1 exhibited essentially zero photobleaching under 8 minutes of continuous excitation with powers similar to those used in the voltage imaging experiments (Fig. $5 \mathrm{~g}$ ), thus supporting recordings of neural activity over behaviorally relevant time scales.

\section{DISCUSSION}

Here we present a methodology for multidimensional directed evolution of proteins via high-throughput screening of large gene libraries in mammalian cells - namely, microscopyguided robotic cell picking. We show that robotic cell picking with single cell precision can be used for directed evolution of proteins, assembling such a system out of commercially available parts that can be installed on any microscope equipped with a motorized stage ${ }^{17}$, and optimizing the software to enable single cell picking according to modularly incorporated image processing algorithms. Since robotic cell screening and selection can be performed on adherent cultured mammalian cells, it enables straightforward interrogation of multiple properties of expressed proteins with easily adjustable spatial and temporal resolutions. These features of our cell picker will enable it to be deployed easily into a wide variety of protein engineering contexts, without requiring the custom fabrication involved with other strategies such as microfluidics ${ }^{5-7}$ or laser-released micropallets ${ }^{36}$. For example, microfluidic devices require custom microfabrication, and can require redesign and reimplementation when screening assays or criteria are changed, which in turn can be challenging for many protein engineers 7,37 . Here, by choosing a strategy that essentially automates the manual steps done by a molecular engineer, and by implementing it with offthe-shelf parts that are easy to set up, and easily customized software, we aim to not only enable powerful multidimensional screens but to democratize the process of performing them.

We used our multidimensional screening approach to generate the opsin-based fluorescent voltage reporter Archon1, which exhibits good membrane localization in neurons of multiple species (mouse, $C$. elegans, zebrafish), severalfold improved brightness over previous opsinbased reporters, severalfold improvements in voltage sensitivity to single APs and in photobleaching over GFP-based reporters, and compatibility with optogenetic control. Our custom cell-picker code allowed us to select cells based on microscopy-obtained quantitative metrics for multiple parameters (i.e., brightness, localization) on multiple cells within a single screening session. The obtained quantitative metrics were then used to identify cells (and variants) possessing an optimal combination of multiple parameters along the Pareto frontier (for our two-dimensional evaluation of brightness and localization) or simply by ranking molecules by the product of normalized parameters (for our three-dimensional evaluation of brightness, localization, and voltage sensitivity) - both attempts to give 
roughly equal priority to each parameter, so that the cells picked reflected molecules simultaneously optimized along multiple axes at once. As a result of this simultaneity, the final variant, Archon1, exhibited improved characteristics for each property selected for, over its precursor template. We demonstrated the utility of Archon1 by imaging single spikes and millivolt-scale subthreshold or synaptic activity in acute mouse brain slices and larval zebrafish in vivo, as well as postsynaptic responses downstream of optogenetic control in $C$. elegans. The ability to survey neural activity in such well-defined systems, e.g., brain circuits from the mouse, or entire transparent organisms, may greatly synergize with new strategies that allow for mapping of physiological data onto fine wiring and connectivity.

Imaging of Archon1 requires excitation light intensity higher than required for GFP-likeprotein-based fluorescent reporters, but the light is red in wavelength and thus less absorbed by tissues than bluer wavelengths. Archon1 supported imaging with about an order of magnitude lower light intensity in comparison to the best performing earlier Arch-based voltage sensors. To achieve light intensity above $0.1 \mathrm{~W} / \mathrm{mm}^{2}$, we here used commercially available red laser diodes, with pricing comparable to LED setups, and found that they provided sufficient light power to image Archon1 in a variety of neural systems as explored in this paper. Thus, in price, such setups might be comparable to blue LED setups used for imaging GCaMP under 1-photon microscopy.

Imaging of neuronal activity using voltage sensors opens up the exciting possibility for simultaneous recordings of large populations of neurons with single cell single spike resolution in vivo. Several ongoing trends, as they mature, will help make voltage imaging even more accessible. Currently available scientific-grade cameras can perform fast imaging (at 500-1000 Hz) over pixel counts smaller by an order of magnitude than those commonly used for calcium imaging (at 10-20 Hz); new cameras capable of fast imaging at cellular resolution over broader fields of view will continue to enhance the power of voltage imaging. Adding in optics capable of large volume imaging with precise optical sectioning (e.g., through light-sheet scanning ${ }^{33,38}$, or through computational optical sectioning ${ }^{39}$ ) may also be of use. Densely labeled neurons may challenge population imaging because assigning photons to individual neurons will be more complex; this may be alleviated by restricting sensor expression to the somata ${ }^{29}$, so that the light emitting sources are made sparser.

\section{ONLINE METHODS}

\section{Molecular cloning and mutagenesis}

The Ace2N-4aa-mNeon, Archer1-KGC-EGFP-ER2 and Quasar2-mOrange-KGC-ER2 genes were synthesized de novo by GenScript, based on the sequences reported in the original publications ${ }^{12,13,16}$. Plasmids encoding mIFP, iRFP670, iRFP682, iRFP702, iRFP and iRFP720 were acquired from Addgene. The $R p \mathrm{BphP1/PAS-GAF}$ domains and QuasAr2ER2 genes were synthesized de novo (Genscript) with mammalian codon optimization and subcloned into the pN1 vector (Clontech) using Agel/NotI sites. Synthetic DNA oligonucleotides used for cloning were purchased from Integrated DNA Technologies. PrimeStar Max mastermix (Clontech) was used for high-fidelity PCR amplifications. Restriction endonucleases were purchased from New England BioLabs and used according 
to the manufacturer's protocols. Ligations were performed using T4 DNA ligase (Fermentas) or InFusion HD kits (Clontech). Small-scale isolation of plasmid DNA was performed with Mini-Prep kits (Qiagen); large-scale DNA plasmid purification was done with GenElute ${ }^{\mathrm{TM}}$ HP Endotoxin-Free Plasmid Maxiprep Kits (Sigma-Aldrich). Random mutagenesis was performed with GeneMorph II Random Mutagenesis Kits (Stratagene), using conditions that resulted in a mutation frequency of up to 15 mutations per 1,000 base pairs. The QuasAr2 random library with a mutation frequency of 10-15 mutations per 1,000 base pairs was prepared by GenScript. Site-directed libraries of Arch mutants were synthesized de novo as gBlocks (EpochLifescience), amplified with corresponding primers using PCR and subcloned into the pN1 vector. Obtained gene libraries in expression vectors were electroporated into NEB10-beta E.coli host cells (New England BioLabs). Serial dilutions $\left(10^{-4}\right.$ and $\left.10^{-5}\right)$ of the electroporated cells were plated on LB/agar medium supplemented with $100 \mathrm{mg} \cdot \mathrm{mL}^{-1}$ kanamycin to estimate electroporation efficiency. The remainder of the cells was grown overnight in LB medium supplemented with $100 \mathrm{mg} \mathrm{mL}^{-1}$ of kanamycin for subsequent plasmid DNA isolation.

To express voltage sensors in primary hippocampal neurons, the corresponding genes were PCR amplified and swapped with the ArchT-GFP gene in pAAV-CaMKIIa-ArchT-GFP plasmid (Addgene plasmid \#37807) using BamHI and EcoRI sites. For in vivo expression in mouse brain the Archon1/2-KGC-EGFP-ER2, QuasAr2-mOrange-KGC-ER2, and Archer1KGC-EGFP-ER2 constructs were cloned into the pCAG-WPRE vector using $K p n I$ and $B S I$ GI sites. For transient expression in zebrafish larvae, we designed the expression vector by cloning the 4 non-repetitive upstream activating sequences (4nrUAS) as previously described ${ }^{41}$ together with a beta actin core minimal promoter followed by the $1.2 \mathrm{~kb}$ long $3^{\prime}$ UTR sequence of Danio rerio synaptotagmin IIa (syt2a). The expression cassette was flanked by Tol2 transposon ends. The Archon-KGC-EGFP-ER2 and miRFP genes were codon-optimized for expression in zebrafish using the online resource at http:// www.bioinformatics.org/, synthesized de novo and cloned into the designed pTol2-4nrUAS vector using SpeI and AscI sites. For expression in C. elegans, a codon-optimized fusion of Archon1 to EGFP (codon optimization was done using the online resource at http:// www.bioinformatics.org/) was subcloned into a pSM vector backbone using KpnI and SacI, and the rig-3 promoter was inserted upstream using FseI and AscI sites.

\section{Protein characterization in vitro}

Protein expression and purification were performed as described previously ${ }^{42}$, with a few modifications. The pBAD/HisB vectors (Life Technologies/Invitrogen) encoding iRFP670, iRFP682, iRFP702, iRFP, iRFP720 and miRFP were co-transformed with pWA23h plasmid, encoding heme oxygenase 1 from Bradyrhizobium ORS278 (hmuO) under the rhamnose promoter, into the BW25113 E.coli strain (CGSC\#7636 in The Coli Genetic Stock Center). Bacterial cells were grown in RM medium supplemented with ampicillin, kanamycin, $0.002 \%$ arabinose, and $0.02 \%$ rhamnose for $15-18 \mathrm{~h}$ at $37^{\circ} \mathrm{C}$ and then for $24 \mathrm{~h}$ at $18^{\circ} \mathrm{C}$. Proteins were purified using TALON Metal Affinity Resin (Clontech) according to the manufacturer's protocol with one minor modification: in the wash buffer, 100mM EDTA was used instead of $400 \mathrm{mM}$ imidazole. The fluorescence spectra were measured using a Fluorolog 3 spectrofluorometer (Jobin Yvon) and a SpectraMax-M5 plate reader (Molecular 
Devices). For absorbance measurements, a Lambda 35 UV/Vis spectrometer (Perkin Elmer) was used. Background light scattering was removed by subtracting a fitted $\lambda^{-4}$ curve from the measured spectrum. For determination of quantum yield, the fluorescence signal of purified proteins was compared with that of the equally absorbing iRFP. To determine the extinction coefficient, we compared the absorbance value for the protein at the main peak centered in the red part of the spectrum with the absorbance value of the short wavelength peak centered at 370-390 nm assuming the latter to have the extinction coefficient of the free biliverdin IXa, which is $39,900 \mathrm{M}^{-1} \mathrm{~cm}^{-1}$ ( $r \mathrm{f}^{43}$ ). $\mathrm{pH}$ titrations were done using a series of commercially available $\mathrm{pH}$ buffers (HYDRION). Size exclusion chromatography was performed by GenScript on a Superdex 200 10/300 GL column (GE Healthcare Life Sciences) using a gel filtration standard (\#1511901; BIO-RAD). Two-photon absorption (2PA) spectra and cross sections of the proteins were measured in PBS buffer, $\mathrm{pH}=7.4$ at concentrations $\sim 1-5 \cdot 10^{-5} \mathrm{M}$ in $1 \mathrm{~mm}$ glass spectroscopy cuvettes (Starna cells) using femtosecond fluorescence. In particular, two-photon excitation (TPE) spectra were collected using an MOM Sutter Instrument two-photon fluorescent microscope coupled with an Insight DeepSee (Newport) femtosecond laser tunable from 680 to $1300 \mathrm{~nm}$. A Plan NeoFluar 2.5x/0.075 Zeiss objective was used to excite and collect fluorescence which was passed through a HQ705/100 filter (Chroma) before reaching the PMT. To correct the TPE spectra for the wavelength-to-wavelength variations of laser properties (pulse duration and beam shape), Styryl 9M (Aldrich) in chloroform was used as a reference standard ${ }^{44}$. The TPE fluorescence had quadratic dependence on excitation power in the whole spectral range as presented in Supplementary Fig. 7. Absolute 2PA cross section was obtained using Styryl 9M (Aldrich) in chloroform as a standard ${ }^{44}$. Fluorescence intensity, $F$, excited at $900 \mathrm{~nm}$, was measured as a function of excitation power, $P$, for both the sample and the reference in the same conditions through a ET675/20 filter (Chroma), with the transmission center at 667 $\mathrm{nm}$ in the MOM setup ( $18^{\circ}$ incidence angle). From the fit of these dependencies to a quadratic function $F=a P^{2}$, a values were obtained and then normalized ${ }^{44}$ to the concentrations (obtained spectrophotometrically, using a BioMate ${ }^{\mathrm{TM}} \mathrm{S} 3$ spectrophotometer) and to the differential quantum efficiencies at $667 \mathrm{~nm}$ (obtained with a spectrofluorimeter, PC1 ISS). In Supplementary Fig. 7, we present the two-photon action spectrum $\left(\sigma_{2} \varphi\right)$ for miRFP and compare it to that of EGFP (measured before ${ }^{45}$ ).

\section{Gene library transfection}

Conventional calcium phosphate transfection was modified to deliver a small number of plasmids per single cell to enable efficient single cell phenotyping and genotyping (Supplementary Fig. 1). HEK293FT cells were selected as the expression host due to several reasons: i) they are suitable for calcium phosphate transfection; ii) they are widely regarded as high expressors for a variety of protein payloads (and excellent for optogenetic tool characterization); iii) they are known to have perhaps the lowest mutation rate among commonly used mammalian cell lines towards exogenous DNA ${ }^{46}$; iv) they are robust and easy to work with. Cells were authenticated by the manufacturer and tested for mycoplasma contamination to their standard levels of stringency and were here used because they are common cell lines for testing new tools. As an expression vector, we used the commercially available pN1 plasmid (Clontech) which can be replicated in HEK293FT cells due to the SV40 ori of replication ${ }^{47}$. The replication of plasmids enables a higher level of protein 
expression upon single copy plasmid delivery, facilitating optical detection of recombinant protein. We used the CMV promoter to drive expression of target genes because it is known to be perhaps the strongest promoter among those commonly used in HEK293 cells ${ }^{48}$.

HEK293FT cells (Invitrogen) were maintained between $10 \%$ and $70 \%$ confluence at $37^{\circ} \mathrm{C}$ with $5 \% \mathrm{CO}_{2}$ in DMEM medium (Cellgro) supplemented with $10 \%$ heat inactivated FBS (Corning), $1 \%$ penicillin/streptomycin (Cellgro), and 1\% sodium pyruvate (BioWhittaker). Transfection of HEK293FT cells with gene libraries was performed using a commercially available calcium phosphate (CaPhos) transfection kit (LifeTechnologies) according to the manufacturer's protocol with minor modifications as follows. HEK293FT cells from the exponential growth phase were seeded at a density to be approximately $70 \%$ confluent on the day of transfection. Culture medium was replaced with fresh medium $\sim 30-60$ minutes before adding DNA-CaPhos co-precipitate so that the medium was of $\mathrm{pH} \sim 7.4 .2 \times$ $\mathrm{CaCl}_{2} / \mathrm{DNA}$ solution was added quickly to an equal volume of $2 \times \mathrm{HBS}$ solution at room temperature, mixed gently for 20-30 sec by pipetting up and down, and added dropwise to the cell culture. Culture medium was carefully replaced with fresh medium 24 hours after transfection. The "empty" pUC19 plasmid was used as "dummy" DNA to keep the total amount of DNA constant for all transfection conditions, and to avoid variation in DNACaPhos co-precipitate formation ${ }^{49,50}$.

\section{FACS screening}

To sort the gene library-transfected HEK293FT cells using flow cytometry, cells were harvested from a culture dish $\sim 48 \mathrm{~h}$ after gene library transfection by applying trypsin for 510 mins (Cellgro) and then washed twice by centrifuging the cell suspension for 5 minutes at $500 \mathrm{rpm}$ and re-suspending cells in PBS (Cellgro). The washed cells were then re-suspended in PBS supplemented with $4 \%$ FBS (Corning) and 10mM EDTA at a density of 1-2.10 cells $/ \mathrm{ml}$ and filtered through a $30 \mu \mathrm{m}$ filter (Falcon) to prevent clogging on the FACS machine. The filtered cells were sorted by FACSAria (BD Biosciences) running BDFACS Diva8.0 software and equipped with standard 405, 488, 561 and $640 \mathrm{~nm}$ solid-state lasers. Debris, dead cells and cell aggregates were gated out using forward and side scatter before desired fluorescence signals were detected (see Supplementary Figure 3a for gating strategy used). For screening $R p B$ phP1/PAS-GAF libraries, excitation at $640 \mathrm{~nm}$ and emission at $670 / 30 \mathrm{~nm}$ and 710/50 nm were used; for QuasAr2 libraries excitation was at $640 \mathrm{~nm}$ and emission was at $710 / 50 \mathrm{~nm}$ (see Supplementary Table 3 for details). Approximately 1.5 times more cells than the size of each library were screened per FACS sorting session and 10-100k cells exhibiting higher fluorescent intensity than that of the positive control (HEK293FT cells transfected with a plasmid encoding the template protein) were collected in a $5 \mathrm{ml}$ tube. Collected cells were plated on a $3 \mathrm{~cm}$ cell culture dish coated with Matrigel (BD Biosciences) for further screening and sorting using our custom cell picker (see below). Sorter cells were analyzed by fluorescence wide-field microscopy to evaluate the abundance of positive cells (on average $80 \%$ of cells were positive).

\section{Multi-parameter screening and single cell isolation using cell picker}

After 24 hour incubation of collected cells in a culture dish, the cell medium was gently replaced with fresh media to remove non-attached cells. Attached cells in the dish were then 
subjected to microscope-guided cell screening using our single cell manipulation system (CellSorter, CellSorter INC) ${ }^{18}$, controlled by software updated for this study to version CellSorter $4.0^{17,18}$. This version was modified from previous versions to be compatible with any microscope, motorized stage, camera and/or other optional hardware (e.g. excitation source) via the open-source micro-manager software (micro-manager.org). In addition, CellSorter4.0 software enables cell analysis, detection, and picking according to external image segmentation and analysis algorithms (in particular, our membrane localization algorithm, described in the section "Protein characterization in mammalian cells"), and acquisition of timelapse movies of selected fields of view (helpful for evaluating fluorophore photostability, which we did on a subset of the cells simultaneously screened on brightness and localization). The "single-mode" mode of operation (see below) that enables picking of one cell per tube, essential for the directed evolution here performed, was also created for the current study. The cell sorter consists of a pulled glass micropipette with an opening of $50 \mu \mathrm{m}$ in diameter, a motorized micromanipulator (Marzhauser SM 3.25), and a pressure controller that manipulates the pressure inside the micropipette. Both the micromanipulator and pressure controller are operated by the CellSorter4.0 software. The cell sorter was installed on an inverted microscope (Nikon Eclipse Ti equipped with 10x NA 0.3, 20x and 40x objective lenses, a SPECTRA-X light engine (Lumencor) with 390/22 nm, 438/24 nm, $475 / 28 \mathrm{~nm}, 510 / 25 \mathrm{~nm}, 585 / 29 \mathrm{~nm}$, and 631/28 nm exciters (Semrock), a 5.5 Zyla camera (Andor), and automated stage (Ludl), controlled by NIS-Elements AR software to obtain fluorescent images of the entire population of cells in a culture dish. To isolate cells with desired properties (e.g. high fluorescence intensity, exclusive plasma membrane localization) from a petri dish, we followed the workflow of cell picking described in Supplementary Figure 2. Briefly, (1) fluorescent images of cells in a culture dish are acquired using the microscope; (2) 10-50 cells exhibiting desired properties (e.g., high fluorescence intensity, exclusive plasma membrane localization) are selected per dish (10-20k cells per $3 \mathrm{~cm}$ dish); (3) the coordinates of selected cells are compiled and fed to the CellSorter software; (4) the CellSorter software orders the micromanipulator to position the tip of the micropipette 5-10 $\mu \mathrm{m}$ above a first target cell; (5) negative pressure is applied through the micropipette to detach and pick up the target cell from the dish; (6) the micropipette moves the cell to a rack where PCR tubes are placed and releases it into a designated PCR tube pre-filled with PBS by applying positive pressure. "Single-mode" (isolation of a single cell per tube) or "multimode" (collection of all cells into a single tube) cell picking can be performed, by selecting the appropriate option in the software.

To simultaneously optimize brightness and localization in a single cell picking step, acquired images were analyzed by custom MATLAB code to quantify brightness (mean fluorescence signal) as well as membrane localization (the ratio of mean fluorescence on the membrane to that of the cytoplasm; cells with ratio $\leq$ were excluded from further analysis for Pareto front identification, since that would mean zero fluorescence enrichment on the membrane vs. cytoplasm), for every detected single cell. Thus, our image analysis reported the coordinates on the dish, as well as two values corresponding to brightness and localization degree, for every detected and analyzed cell. These quantitative metrics were then used to identify cells with optimal combinations of both brightness and membrane localization via the concept of Pareto optimality. Briefly, we first identified the cell with the highest 
brightness, and the cell with the best localization. Then, we identified a set of cells that have at least one parameter better than its corresponding parameter for the aforementioned pair of cells, in a fashion so that there are no cells outside this set that would have simultaneously both parameters better than any cells within the identified set. Next, we counted the number of cells in the identified set plus the first pair of identified cells. If this number was lower than number of cells we wanted to pick, we repeated this selection calculation on the set of the detected cells, excluding the set of cells already selected in the first iteration, until the total number of cells identified equals the number of cells we wanted to pick. In turn, the number of cells we wanted to pick was defined by the number of clones we thought we could feasibly screen after gene recovery.

\section{Target gene recovery}

Cells individually collected in PCR tubes by the cell picker were subjected to whole genome amplification using a commercially available whole genome amplification kit (WGA, New England BioLabs) followed PCR amplification. Amplicons with a size corresponding to that of the target gene were purified by agarose gel electrophoresis and cloned into an expression vector, and the purified plasmids were individually transfected and expressed in HEK cells for assessing desired characteristics of each gene. To account for the mutagenic activity of HEK293T cells towards exogenous DNA and potential incorporation of multiple plasmids into a given cell (see Supplementary Fig. 1 for details), at least 5 genes were screened from each HEK cell isolated.

\section{Protein characterization in mammalian cells}

HEK293FT (Invitrogen) and HeLa (ATCC CCL-2) cells were maintained between 10\% and $70 \%$ confluence at $37^{\circ} \mathrm{C}$ with $5 \% \mathrm{CO} 2$ in DMEM medium (Cellgro) supplemented with $10 \%$ heat inactivated FBS (Corning), 1\% penicillin/streptomycin (Cellgro), and 1\% sodium pyruvate (BioWhittaker). Cells were authenticated by the manufacturer and tested for mycoplasma contamination to their standard levels of stringency and were here used because they are common cell lines for testing new tools. We used HeLa cells simply as a testbed for protein expression, and not for any reasons of investigating the properties of HeLa cells in their own right. HEK293FT and HeLa cells were transiently transfected using TransIT-X2 (Mirus Bio LLC) according to the manufacturer's protocol and analyzed $48 \mathrm{~h}$ after transfection. Cells were imaged using a Nikon Eclipse Ti inverted microscope equipped with a SPECTRA X light engine (Lumencor) with 475/28 nm and 631/28 nm exciters (Semrock), and a 5.5 Zyla camera (Andor), controlled by NIS-Elements AR software, and using 10x NA 0.3 (Supplementary Fig. 3c, 4b,c) and 40x NA 1.15 (Fig. 1c,d and Supplementary Fig. 3d, $4 \mathrm{~d}, 6,10 \mathrm{~b}, \mathrm{c})$ objective lenses. To compare brightness of mIFP, miRFP and $R p \mathrm{BphP} 1$ intermediate mutants we calculated the mean near-infrared fluorescence intensity of $\sim 100 \%$ confluent HEK293FT cell cultures expressing corresponding proteins (Supplementary Fig. $4 \mathrm{~b}, \mathrm{c})$. For plasma membrane localization analysis, voltage sensor variants were cotransfected with membrane-anchored YFP (Fig. 1d, Supplementary Fig. 10b). To quantify protein localization, we wrote MATLAB code that automatically detected cells in each image and calculated the degree of similarity (i.e., co-localization) between the normalized images acquired in the green and red channels by averaging the difference between the green channel intensity profile and the red channel intensity profile, and then taking the reciprocal 
of this ratio (so that a higher value means better co-localization). For Supplementary Fig. 4d, raw photobleaching curves were normalized to the spectrum of the red LED of SPECTRA X light engine, the transmission profile of the excitation filter and dichroic mirror, and the absorbance spectrum of respective FP. For flow cytometry analysis HEK293T cells were stained with SYTOX Green (Life Technologies) and analyzed using 488 and $640 \mathrm{~nm}$ laser lines and the respective 515/20BP (BP, bandpass) and 710/50BP emission filters on a BD LSR II analyzer (Fig. 1e and Supplementary Fig. 10a).

\section{Induced transmembrane voltage (ITV) in HEK cells}

To screen for voltage sensitivity, HEK293FT cells expressing mutants selected with our cell picker system were subjected to a reproducible electric field between two platinum electrodes as described previously ${ }^{12,51}$ with two minor modifications. First, to measure the actual value of the electric field applied across the cell culture environment, we used an oscilloscope (Agilent Technologies) connected in parallel to the platinum electrodes deployed. Second, to increase the throughput of ITV screening, the positions of the platinum electrodes were controlled by the CellSorter micromanipulator and associated software. In brief, HEK293FT cells were plated on 24 well plates and transfected with $500 \mathrm{ng}$ of target plasmid DNA per well using TransIT-X2 (Mirus Bio LLC) following the manufacturer's protocol. Cell imaging was performed on an inverted Eclipse Ti-E (Nikon) equipped with a CMOS camera (Zyla5.5, Andor), LEDs (Spectra, Lumencor), a $637 \mathrm{~nm}$ Laser (637 LX, OBIS) focused on the back focal plane of a 40x NA 1.15 objective (Nikon), and a filter set with 664LP (LP, longpass; emission) and 650nm (dichroic) filters (Semrock). The pair of platinum electrodes, with a gap of $4 \mathrm{~mm}$, and mounted on our automated micromanipulator, was sequentially placed in the wells, and trains of electrical pulses $(20-100 \mathrm{~V} / \mathrm{cm}, 50 \mathrm{~ms}, 2$ Hz) generated by a DG2041A Arbitrary Waveform Function Generator (RIGOL) and amplified with a high voltage amplifier (Model 2205, Trek) were applied across the cell culture to induce changes in the membrane voltage. Fluorescent images were recorded at a $200 \mathrm{~Hz}$ frame rate in $2 \times 2$ binning mode for $20 \mathrm{~s}$.

\section{Whole-cell electrophysiology and fluorescence recording in HEK cells}

Voltage sensitive variants selected from the ITV screening were subjected to whole-cell electrophysiology in HEK293FT cells. To evaluate voltage sensor candidates, HEK293FT cells were transfected with $100 \mathrm{ng}$ of target plasmid DNA using the calcium phosphate protocol described above. 24 hours post transfection, HEK293FT cells were re-plated on round coverslips $(0.15 \mathrm{~mm}$ thick, $25 \mathrm{~mm}$ in diameter, coated with $2 \%$ growth factor reduced Matrigel in DMEM for $1 \mathrm{~h}$ at $37^{\circ} \mathrm{C}$ ) at a density of 20,000 cells per well in a 24-well plate and incubated for a day at $37^{\circ} \mathrm{C}$. Whole-cell patch clamp recording was performed between 48 and $72 \mathrm{~h}$ post transfection in Tyrode's solution consisting of (in $\mathrm{mM}$ ) $125 \mathrm{NaCl}, 2 \mathrm{KCl}, 3$ $\mathrm{CaCl}_{2}, 1 \mathrm{MgCl}_{2}, 10 \mathrm{HEPES}, 30$ glucose, $\mathrm{pH} 7.3$ (NaOH adjusted) at $320 \mathrm{mOsm}$; the intracellular solution consisted of (in mM) $135 \mathrm{~K}$-gluconate, $8 \mathrm{NaCl}, 10 \mathrm{HEPES}, 4 \mathrm{Mg}$-ATP, $0.4 \mathrm{Na}-\mathrm{GTP}, 0.6 \mathrm{MgCl}_{2}, 0.1 \mathrm{CaCl}_{2}$, pH 7.25 (KOH adjusted) at 295mOsm. A gap-junction blocker, 2-aminoethoxydiphenyl borate ( $50 \mu \mathrm{M}$, Sigma), was added to eliminate electrical coupling between cells. All-trans-retinal was not supplemented for any HEK cell recordings. Borosilicate glass pipettes (WPI) with an outer diameter of $1 \mathrm{~mm}$ and a wall thickness of 0.2 mm were pulled using a Flaming/Brown micropipette puller (P-97, Sutter Instruments) to 
obtain a tip resistance of 3-10 M $\Omega$. Pipettes were positioned by a Sutter MP285 manipulator during whole-cell patching. To ensure accurate measurements, data was acquired from HEK293FT cells with access resistance $<15 \mathrm{M} \Omega$, having resting potentials between -10 and $-40 \mathrm{mV}$, membrane resistance $>0.3 \mathrm{G} \Omega$, and holding current (for a holding potential of -70 $\mathrm{mV}$ ) within $\pm 100 \mathrm{pA}$. For Fig. 1f,g and Supplementary Figure 10, patch-clamp recordings were acquired via an Axopatch 700B amplifier (Molecular Devices) and Digidata 1440 digitizer (Molecular Devices) in Tyrode's solution maintained at $32^{\circ} \mathrm{C}$ during experiments using a warmed holding platform (64-1663D, Warner Instruments) controlled by a temperature controller (TC-324B, Warner Instruments). Fluorescence imaging was performed on an inverted fluorescence microscope (Nikon Ti), equipped with a red laser (637 nm, $100 \mathrm{~mW}$, Coherent, OBIS 637LX, pigtailed) expanded by a beam expander (Thorlabs) and focused onto the back focal plane of a 40x NA 1.15 objective lens (Nikon). Images were taken by an EMCCD camera (iXon, Andor) at a $500 \mathrm{~Hz}$ frame rate in $2 \times 2$ binning mode for $2 \mathrm{~s}$. The voltage sensitivity was imaged in voltage-clamp mode with a holding potential of $-70 \mathrm{mV}$ for $1 \mathrm{~s}$ and then applying voltage steps from $-70 \mathrm{mV}$ to $+30 \mathrm{mV}$ for $100 \mathrm{~ms}$. For Supplementary Figure 17, photocurrents were recorded at room temperature in voltage-clamp mode with a holding potential of $-70 \mathrm{mV}$ in response to $500 \mathrm{~ms}$ light pulses using a Multiclamp 700B and Digidata 1550A digitizer (Molecular Devices), and a PC running pClamp10 (Molecular Devices).

\section{Primary neuron culture and transfection}

All procedures involving animals at MIT were conducted in accordance with the US National Institutes of Health Guide for the Care and Use of Laboratory Animals and approved by the Massachusetts Institute of Technology Committee on Animal Care. Hippocampal neurons were prepared from postnatal day 0 or 1 Swiss Webster (Taconic) mice (both male and female mice were used) as previously described ${ }^{4,52}$ with the following modifications: dissected hippocampal tissue was digested with 50 units of papain (Worthington Biochem) for 6-8 min, and the digestion was stopped with ovomucoid trypsin inhibitor (Worthington Biochem). Cells were plated at a density of 20,000-30,000 per glass coverslip coated with Matrigel (BD Biosciences). Neurons were seeded in 100 $\mu$ l plating medium containing MEM (Life Technologies), glucose (33mM, Sigma), transferrin $(0.01 \%$, Sigma), Hepes (10mM, Sigma), Glutagro (2mM, Corning), Insulin (0.13\%, Millipore), B27 supplement (2\%, Gibco), and heat inactivated FBS (7.5\%, Corning). After cell adhesion, additional plating medium was added. AraC $(0.002 \mathrm{mM}$, Sigma) was added when glia density was $50-70 \%$ of confluence. Neurons were grown at $37^{\circ} \mathrm{C}$ and $5 \% \mathrm{CO}_{2}$ in a humidified atmosphere.

Cultured neurons were transfected at 4-5 days in vitro (DIV) with a commercial calcium phosphate transfection kit (Life Technologies) as previously described ${ }^{4}$. Briefly, $500 \mathrm{ng}$ of plasmid DNA per well was used for transfection followed by additional washing with acidic MEM buffer (pH 6.7-6.8) after 30-60 min of calcium phosphate precipitate incubation to remove residual precipitates ${ }^{53}$. All measurements on neurons were taken between DIV 14 and 18 DIV ( $~ 9-14 \mathrm{~d}$ post transfection) to allow for sodium channel maturation. No alltrans-retinal was supplemented for any cultured neuron recordings. 


\section{Fluorescence microscopy of primary neurons}

Fluorescent imaging of voltage sensors expressed in cultured hippocampal neurons for Fig. 2, Supplementary Fig. 12-16 and Supplementary Fig. 18, 19 was performed using a Nikon Eclipse Ti inverted microscope equipped with a 40x NA 1.15 water immersion objective (Nikon), a $637 \mathrm{~nm}$ Laser (637 LX, OBIS) focused on the back focal plane of the objective, a SPECTRA X light engine (Lumencor) with $475 / 28 \mathrm{~nm}, 585 / 29 \mathrm{~nm}$, and 631/28 nm exciters (Semrock), a $470 \mathrm{~nm}$ LED (ThorLabs) and a 5.5 Zyla camera (Andor), controlled by NISElements AR software.

\section{Electrophysiology in primary hippocampal neurons}

Whole-cell patch clamp recordings of cultured neurons for Fig. 2 and Supplementary Fig. 14-16 were acquired via an Axopatch 700B amplifier (Molecular Devices) and Digidata 1440 digitizer (Molecular Devices). Neurons were patched between DIV14 and DIV18. Neurons were bathed in Tyrode's solution ( $125 \mathrm{NaCl}, 2 \mathrm{KCl}, 3 \mathrm{CaCl} 2,1 \mathrm{MgCl} 2,10 \mathrm{HEPES}$, 30 glucose, $\mathrm{pH} 7.3(\mathrm{NaOH}$ adjusted) $)$ at $32^{\circ} \mathrm{C}$ during measurements. Borosilicate glass pipettes with an outer diameter of $1 \mathrm{~mm}$ and a wall thickness of $0.2 \mathrm{~mm}$ with resistance of 3$10 \mathrm{M} \Omega$ were filled with internal solution containing $135 \mathrm{~K}$-gluconate, $8 \mathrm{NaCl}, 10 \mathrm{HEPES}, 4$ Mg-ATP, 0.4 Na-GTP, 0.6 MgCl2, 0.1 CaCl2, pH 7.25 (KOH adjusted) at 295 mOsm. Measurements from primary neuron cultures were performed on the electrophysiology setup described in the "Whole-cell electrophysiology and fluorescence recording in HEK cells" section. Patch-clamp data was acquired only if the resting potential was below $-45 \mathrm{mV}$ and access resistance was $<25 \mathrm{M} \Omega$. Access resistance was compensated at 30-70\%. Fluorescence imaging was performed on an inverted fluorescence microscope (Nikon Ti), equipped with a red laser (637nm, 100mV, Coherent, OBIS 637LX, Pigtailed) expanded by a beam expander (Thorlabs) and focused onto the back focal plane of a 40x NA 1.15 objective lens (Nikon). Synaptic blockers (NBQX, $10 \mu \mathrm{M}$; d(-)-2-amino-5-phosphonovaleric acid, $25 \mu \mathrm{M}$; gabazine, $20 \mu \mathrm{M}$; Tocris) were added to the imaging medium for measurements of singlecell electrophysiology. For voltage sensor kinetics studies presented in Fig. 2c,

Supplementary Fig. 15,16 and Supplementary Table 1 images were acquired with an EMCCD camera (iXon, Andor) at a $3.2 \mathrm{kHz}$ frame rate using an optical mask (Optomask, Andor). For other concurrent imaging and electrophysiology recordings the acquisition rate was reduced to $2.3 \mathrm{kHz}$ to achieve longer recording times without camera overheating and to reduce data storage.

\section{In utero electroporation}

Embryonic day (E) 15.5 timed-pregnant female C57BL/6 and CD1 (Charles River; for Fig. 3, Supplementary Figs. 20-22, 24, and 25) or Swiss Webster (Taconic; Supplementary Fig. 7,23 ) mice were deeply anesthetized with $2 \%$ isoflurane. Uterine horns were exposed and periodically rinsed with warm sterile PBS. A plasmid encoding Archon1, Archon2 or miRFP (pCAG-Archon1/2-KGC-EGFP-ER2-WPRE, pAAV-Syn-miRFP; $1 \mu \mathrm{g} / \mu \mathrm{l}$ ) diluted with PBS was injected into the lateral ventricle of one cerebral hemisphere of an embryo. Five voltage pulses $(50 \mathrm{~V}, 50 \mathrm{~ms}$ duration, $1 \mathrm{~Hz})$ were delivered using round plate electrodes (CUY21 electroporator, NEPA GENE, Japan; ECM $^{\mathrm{TM}} 830$ electroporator, Harvard Apparatus). Injected embryos were placed back into the dam, and allowed to mature to 
delivery. All experimental manipulations were performed in accordance with protocols approved by the Harvard Standing Committee on Animal Care or Massachusetts Institute of Technology Committee on Animal Care (according to location of the respective experiments), following guidelines described in the US National Institutes of Health Guide for the Care and Use of Laboratory Animals.

\section{Acute brain slice preparation}

Acute brain slices were obtained from C57BL/6 and CD1 (Charles River) mice at P20 P30, using standard techniques. Mice were used without regard for sex. No statistical methods were used to estimate sample size for animal studies throughout. No randomization or blinding were used for animal studies throughout. Mice were anaesthetized by isoflurane inhalation and perfused transcardially with ice-cold artificial cerebrospinal fluid (ACSF) containing (in mM) $125 \mathrm{NaCl}, 2.5 \mathrm{KCl}, 25 \mathrm{NaHCO}_{3}, 2 \mathrm{CaCl}_{2}, 1 \mathrm{MgCl}_{2}, 1.25 \mathrm{NaH}_{2} \mathrm{PO}_{4}$ and 11 glucose $(295 \mathrm{mOsm} / \mathrm{kg})$. Cerebral hemispheres were removed, placed in cold cholinebased cutting solution (consisting of (in $\mathrm{mM}$ ): 110 choline chloride, $25 \mathrm{NaHCO}_{3}, 2.5 \mathrm{KCl}, 7$ $\mathrm{MgCl}_{2}, 0.5 \mathrm{CaCl}_{2}, 1.25 \mathrm{NaH}_{2} \mathrm{PO}_{4}, 25$ glucose, 11.6 ascorbic acid, and 3.1 pyruvic acid), blocked and transferred into a slicing chamber containing ice-cold choline-based cutting solution. Coronal slices ( $300 \mu \mathrm{m}$ thick) were cut with a Leica VT1000s vibratome, transferred to a holding chamber containing ACSF, and recovered for $30 \mathrm{~min}$ at $34^{\circ} \mathrm{C}$, followed by another $30 \mathrm{~min}$ at room temperature. Slices were subsequently maintained at room temperature until use. Both cutting solution and ACSF were constantly bubbled with $95 \% \mathrm{O}_{2} / 5 \% \mathrm{CO}_{2}$.

\section{Electrophysiology and voltage imaging in acute brain slice}

Individual slices were transferred to a recording chamber mounted on an upright microscope (Olympus BX51WI, see below) and continuously superfused ( $2-3 \mathrm{ml} / \mathrm{min}$ ) with ACSF warmed to $\sim 32^{\circ} \mathrm{C}$ by passing it through a feedback-controlled in-line heater (SH-27B; Warner Instruments). Cells were visualized through a $60 \mathrm{x}$ water-immersion objective with either infrared differential interference contrast (DIC) optics, or epifluorescence to identify GFP-positive cells. Whole-cell voltage- and current-clamp recordings were obtained from GFP-positive pyramidal neurons in layer $2 / 3$ of motor cortex, using patch pipettes (tip resistance 2.2-3.5 M $\Omega$ ) pulled from borosilicate glass (G150F-3, Warner Instruments). For current-clamp recordings the pipette solution consisted of in (mM): $130 \mathrm{~K}$-gluconate, 10 $\mathrm{KCl}, 4 \mathrm{NaCl}$, 10 HEPES, $4 \mathrm{Mg}_{2}$-ATP, 0.3 Tris-GTP, 14 Tris-phosphocreatine (290 mOsm/l; $\mathrm{pH} 7.28$ adjusted with $\mathrm{KOH}$ ), and for voltage-clamp recordings a cesium-based pipette solution was used (135 $\mathrm{CsMeSO}_{3}, 1 \mathrm{EGTA}(\mathrm{CsOH}), 10 \mathrm{HEPES}, 3.3 \mathrm{QX}-314(\mathrm{Cl}-)$, $4 \mathrm{Mg}_{2}-$ ATP, 0.3 Na-GTP, $\mathrm{Na}_{2}$-phosphocreatine; $295 \mathrm{mOsm} / \mathrm{l}$; pH 7.35 adjusted with $\mathrm{CsOH}$ ). For 2photon imaging of recorded cells $20 \mu \mathrm{M}$ AlexaFluor594 was added to the respective internal solution. Voltage-clamp recordings were performed in the presence of tetrodotoxin (TTX, $0.5 \mu \mathrm{M})$ and cadmium $(50 \mu \mathrm{M})$. EPSPs were evoked by positioning a tungsten bipolar electrode (FHC) in layer 5 , and delivering a train of 5 pulses $(0.1 \mathrm{~ms}, 1 \mathrm{~Hz})$. Individual trials were separated by $>30$ s. Stimulation strength was adjusted to evoke sub-threshold EPSPs, and only cells in which clean, short-latency EPSPs could be evoked were used for voltage imaging. 
Archon fluorescence was excited via a red laser $(637 \mathrm{~nm}, 140 \mathrm{~mW}$, Coherent Obis 637-140 LX), which was focused onto the back focal plane of the objective (Olympus LUMFL N $60 \mathrm{x} / 1.10 \mathrm{~W})$. Neutral density filters were used such that the power recorded after the objective was $\sim 7 \mathrm{~mW}$. The laser spot was $\sim 25 \mu \mathrm{m}$ in diameter at the sample plane; the resulting intensity was $\sim 15 \mathrm{~W} / \mathrm{mm}^{2}$. Fluorescence was collected through the same objective, passed through a 705/100 nm emission filter, and imaged onto an EMCCD camera (Andor iXON Ultra 888) at 1000 frames/s. In order to acquire images at this frame rate, we restricted the EMCCD region of interest to a 99 x 300 pixel window (binned $3 \times 3$ ), and individual sweeps were no longer than $30 \mathrm{~s}$. Membrane currents and potentials were amplified and low-pass filtered at $3 \mathrm{kHz}$ using a Multiclamp 700B amplifier (Molecular Devices), digitized at $10 \mathrm{kHz}$ and acquired using National Instruments acquisition boards and a custom version of ScanImage written in MATLAB (Mathworks) (https://github.com/ bernardosabatinilab/SabalabSoftware_Nov2009.git). For two-photon images presented in Supplementary Fig. 22, individual neurons expressing Archon1 were filled through a recording pipette with an internal solution containing Alexa Fluor $594(20 \mu \mathrm{M})$, and both Alexa Fluor 594 and GFP fluorescence were visualized using a Ti-Sapphire laser (Coherent) tuned to $850 \mathrm{~nm}$.

\section{Visualization of Archon expression in fixed brain tissue}

Deeply anesthetized mice were perfused transcardially with $4 \%$ paraformaldehyde in $0.1 \mathrm{M}$ phosphate buffer ( $\mathrm{pH} 7.3)$ and brains were postfixed for $4 \mathrm{~h}$ at $4^{\circ} \mathrm{C} .50 \mu \mathrm{m}$ sections were cut on a vibratome, stained with fluorescent Nissl stain (NeuroTrace 640/660; Molecular Probes), and mounted in ProLong Antifade Diamond reagent (Invitrogen). Images were taken first with a slide-scanning wide-field microscope (VS120, Olympus), and highresolution images of regions of interest were subsequently acquired with a Leica LS8 confocal microscope (Harvard NeuroDiscovery Center). Confocal images represent maximum intensity projections of 15 to $40 \mu \mathrm{m}$ image stacks.

\section{Transient expression in zebrafish embryos}

All experiments were conducted in accordance with MIT Committee on Animal Care and Janelia Farm IACUC protocols (as appropriate for the respective locations of specific experiments). Zebrafish, Danio rerio species, were raised and bred at $28^{\circ} \mathrm{C}$ according to standard methods. DNA plasmids encoding zArchon1-KGC-GFP-ER2 or miRFP were coinjected with Tol2 transposase mRNA into embryos of the pan-neuronal expressing Gal4 line, $\operatorname{tg}(\text { elav13:GAL4-VP16 })^{\text {nns }}\left({ }_{(r e f}{ }^{5}\right)$. The embryos used in the study were homozygous nacre. Briefly, DNA and Tol2 transposase mRNA, synthesized using pCR2FA as a template $\left(r e f^{55}\right)$ (mMESSAGE mMACHINE ${ }^{\circledR}$ SP6 Transcription Kit, Thermofisher), were diluted to a final concentration of $25 \mathrm{ng} / \mu \mathrm{l}$ in $0.4 \mathrm{mM} \mathrm{KCl}$ solution containing $0.05 \%$ phenol red solution (Sigma Aldrich) to monitor the injection quality. The mixture was kept on ice to minimize degradation of mRNA during the injection. The mixture was injected into embryos at $1-4$ cell stages as described previously ${ }^{56}$. Larvae, $\operatorname{tg}(\text { elavl3:GAL4-VP16 })^{n n s}{ }_{(}\left(\mathrm{ref}^{54}\right)$, were screened for green fluorescence in the brain and spinal cord at 2-3 days post fertilization (dpf; animals were used without regard to sex) and subsequently imaged at 3-4 dpf. 


\section{Voltage imaging in zebrafish embryos}

Zebrafish larvae at 3-4 dpf were used to image neurons expressing zArchon1. To prevent motion artifacts during imaging, larvae were paralyzed by applying a paralytic agent, pancronium bromide, at a final concentration of $0.20 \mathrm{mg} / \mathrm{ml}$ (Sigma Aldrich), to stop muscle motion. Larvae were placed in a dish with the paralytic agent until they stopped moving, taking about one minute on average. Paralyzed larvae were immobilized in 1.5\% ultra-lowmelting agarose (Sigma Aldrich) prepared in E3 medium following standard protocols ${ }^{57}$. The embedded larvae were mounted on an inverted epifluorescent microscope (Nikon Eclipse $T$ ) for voltage imaging. The fluorescence of zArchons was excited by a red laser (OBIS 637 LX) focused onto the back focal plane of a 40x 1.15NA water-immersion objective (Nikon). Illumination intensities of $1.1-2.2 \mathrm{~W} / \mathrm{mm}^{2}$ were used for voltage imaging recorded using a sCMOS camera (Zyla 5.5, Andor) with image acquisition rates of $250-$ $500 \mathrm{~Hz}$. No chemical or physical stimuli were applied before or during recording of spontaneous activity.

\section{Lightsheet zebrafish imaging}

Lightsheet imaging for Supplementary Fig. 7 was performed on a Zeiss Z.1 lightsheet microscope. The fixed sample was embedded in $1 \%$ agarose in a capillary and mounted on the freely rotating stage of the Z.1 lightsheet microscope. For image acquisition, the sample with the surrounding agarose gel was extruded from the glass capillary. Lightsheets were generated by two illumination objectives (10x, NA 0.2), and the fluorescence signal detected by a 20x water immersion objective (NA 1.0). The laser line used for excitation was $638 \mathrm{~nm}$. Optical filters used to separate and clean the fluorescence response included a Chroma T647lpxr as a dichroic, and a Chroma ET665lp for miRFP. Tiled datasets were taken with the Zeiss ZEN Software, and subsequently merged and processed with FIJI, and Arivis Vision4D.

\section{Voltage imaging in C. elegans}

The Archon1-KGC-EGFP-ER2 gene was codon-optimized for expression in C. elegans using the online resource at http://www.bioinformatics.org/. Worms were maintained and grown following standard protocols ${ }^{58}$. SWF4 (flvEx 3[rig-3:: wArchon1-KGC-EGFP-ER2, sra-6::ChR2-GFP, elt-2::nGFP]) and SWF5 (flvEx4[rig-3::wArchon1-KGC-EGFP-ER2, sra-6::ChR2-GFP), elt-2::nGFP]) were two independent lines generated by injecting the indicated plasmids into $\mathrm{N} 2$ background worms and picking those with strongest expression of the wArchon1-EGFP fusion. Results from these two lines were indistinguishable. SWF7 (flvEx S[rig-3::WArchon1-KGC-EGFP-ER2, elt-2::nGFP]), generated by injecting the indicated plasmid into $\mathrm{N} 2$ background worms, was used for control experiments examining the action of wArchon1 in the absence of channelrhodopsin-2.

The SWF4, SWF5 and SWF7 transgenic worms (used without regard to sex) at L4 stage of development were put onto NGM plates with OP50 lawns supplemented with $100 \mu \mathrm{M}$ alltrans-retinal (Sigma-Aldrich, USA) no less than 16 hours prior to experiments. Worms were mounted on 5\% agarose pads on microscope slides, immobilized with $5 \mathrm{mM}$ tetramisole and imaged using a Nikon Eclipse Ti inverted microscope equipped with a 40x NA 1.15 water immersion objective (Nikon), a 637nm Laser (637 LX, OBIS) focused on the back focal 
plane of the objective, a SPECTRA X light engine (Lumencor) with 475/28 nm excitation filter (Semrock), and a 5.5 Zyla camera (Andor), controlled by NIS-Elements AR software. Fluorescence of wArchon 1 was imaged with $635 \mathrm{~nm}$ excitation at $800 \mathrm{~mW} / \mathrm{mm}^{2}$ and $664 \mathrm{LP}$ emission filter (Semrock); GFP fluorescence was imaged with a 475/34BP excitation filter and a 527/50BP emission filter (Semrock). Optogenetic stimulation was performed with $475 / 34 \mathrm{~nm}$ illumination at $0.2 \mathrm{~mW} / \mathrm{mm}^{2}$.

\section{Data analysis and statistics}

Data were analyzed offline using NIS-Elements Advance Research software, Origin (OriginLab), c, Excel (Microsoft), ImageJ, Igor Pro (Wavemetrics), BoxPlotR and MATLAB. Analysis of all presented fluorescence traces was performed as following: cells and a neighboring cell-free region were selected manually and fluorescence measurements were performed for each region of interest (ROI), and then fluorescence from an Archonfree region was subtracted from cell fluorescence to correct for background; except Fig. 3 and Supplementary Fig. 24, 25, in which Archon fluorescence was extracted by a maximumlikelihood pixel-weighting algorithm described previously ${ }^{59}$. In addition, for Fig. 3 and Supplementary Fig. 24, 25 Archon fluorescence traces were corrected for photobleaching by subtracting baseline fluorescence traces that were low-pass filtered and fit to a double exponential or an exponential function. All fluorescent traces were presented without noise filtering except for the zoomed-in trace in Fig. 4c (bottom), which was filtered for noise using a moving average window. Fluorescence changes to voltage steps were calculated as $\Delta \mathrm{F} / \mathrm{F}=\left(\mathrm{F}_{\mathrm{ss}}-\mathrm{F}_{\mathrm{bl}}\right) / \mathrm{F}_{\mathrm{bl}}$, where $\mathrm{F}_{\mathrm{ss}}$ (steady-state fluorescence) is the mean fluorescence intensity averaged over 50-70 ms during a voltage step after the fluorescence signal reaches its plateau, and $\mathrm{F}_{\mathrm{bl}}$ (baseline fluorescence) is the mean fluorescence intensity averaged over 100 $\mathrm{ms}$ before the voltage step. Fluorescence changes during the action potential (AP) were calculated as $\Delta F / F=\left(F_{\text {peak }}-F_{b 1}\right) / F_{\text {bl }}$, where $F_{\text {peak }}$ (peak fluorescence) is the max fluorescence intensity during an $\mathrm{AP}, \mathrm{F}_{\mathrm{bl}}$ (baseline fluorescence) is the mean fluorescence intensity averaged over the 100 to $200 \mathrm{~ms}$ before an AP. The SNR for an AP was calculated by dividing the peak fluorescence of an AP by the standard deviation of the baseline fluorescence over a 100 to $200 \mathrm{~ms}$ window preceding the AP. These SNRs were averaged to determine the AP SNR for a given cell. AP width was calculated at $50 \%$ of peak AP fluorescence by linearly interpolating the average AP fluorescence for a cell. This width was compared to the electrical AP waveform width after the electrical signal was down-sampled to the frame rate of the camera.

For kinetics analysis (Fig. 2c, Supplementary Fig. 10, 16, 24, 25, and Supplementary Table $1,6)$, fluorescent traces were averaged across cells and the fluorescence rise segment and fluorescence decay segment were extracted from the averaged trace in MATLAB by inspection. Only the first $50 \mathrm{~ms}$ in the fluorescence rise and fluorescence decay segments were used in the downstream bi-exponential fitting. Next, the fluorescence rise (inverted for convenience of using the single equation below for both rise and decay) or decay segment, $\mathrm{F}(\mathrm{t})$, was fitted to the following bi-exponential function in MATLAB: $\mathrm{F}(\mathrm{t})=\mathrm{A} \times(\mathrm{C} \times \exp (-\mathrm{t} /$ $\left.\left.t_{1}\right)+(1-C) \times \exp \left(-t / t_{2}\right)\right)$, where $t_{1}$ was the time constant of the fast component and $t_{2}$ was the time constant of the slow component. The percentage of the total magnitude that was associated with the fast component $\left(\% \mathrm{t}_{1}\right)$ was defined as " $\mathrm{C}$ " above. For Supplementary Fig. 
24,25 , to measure the time constant of actual voltage changes in voltage-clamp experiments, a series of hyperpolarizing voltage steps ( 6 repetitions of $-5 \mathrm{mV}$ ) were applied immediately before the voltage imaging protocol (without capacitance and series resistance compensation), and the decay constants of the first transient of each step was analyzed in the same way as described above.

For Supplementary Fig. 19e-f, ROIs for the Archon 2 signal were identified using a novel algorithmic approach utilizing non-negative matrix factorization $(\mathrm{NMF})^{60}$ on the power spectral density of each pixel's time history. Intuitively, pixels that do not represent Archon2 activity will have a time history that is a mix of noise and camera artifacts, whereas pixels that do capture Archon2 activity will have a distinct signature in the frequency domain that captures the Archon 2 dynamics. To automatically separate both the spatial and time history of these two types of pixels, a rank-2 NMF is calculated on the 3D dataset (X, Y and frequency), reshaped as a 2D matrix (space and frequency). What is required from a human is to specify one pixel that is known to be demonstrating Archon2 signal. For all other pixels, the algorithm compares the weight of the NMF component known to correspond to noise versus the NMF component known to correspond to Archon2 activity. For robustness, this algorithm is applied to overlapping partitions of the data, and then each pixel receives a set of votes as either noise or Archon 2 signal. The result of this voting system is a mask that can applied to the entire dataset, removing $98 \%$ of the pixels. The remaining data is then spatially clustered via connected components and available for existing time-domain interrogations. The code is available as a MATLAB script at https:/github.com/ dgoodwin208/nmfroi.

All statistics were performed in JMP (SAS), except that Wilcoxon rank sum tests were performed in MATLAB (MathWorks). The Wilcoxon rank sum test was used for comparing two groups. The Kruskal-Wallis test was used for comparing multiple groups. For the Kruskal-Wallis test, Steel's post hoc test was used to compare each group to a control group; Steel-Dwass' post hoc test was used to compare each pair of groups. We did not perform a power analysis, since our goal was to create a new technology; as noted in ref. ${ }^{61}$, and recommended by the NIH, "In experiments based on the success or failure of a desired goal, the number of animals required is difficult to estimate..." As noted in the aforementioned paper, "The number of animals required is usually estimated by experience instead of by any formal statistical calculation, although the procedures will be terminated [when the goal is achieved].” These numbers reflect our past experience in developing neurotechnologies.

All attempts at replication of the experiments were successful.

\section{Data availability}

The data that support the findings of this study are available from the corresponding author on request. Sequences of the reported proteins are available at Genbank at the following accession codes: miRFP MG250278; zmiRFP, MG250279; Archon1, MG250280; Archon2, MG250281; zArchon1, MG250282; zArchon2, MG250283; wArchon1, MG250284; wArchon2, MG250285. All genes of interest will be posted to Addgene for distribution upon publication. 


\section{Code availability}

Computer code used to generate results for this study are available from the corresponding author on request.

\section{Supplementary Material}

Refer to Web version on PubMed Central for supplementary material.

\section{Acknowledgments}

We thank G. Paradis and M. Saturno-Condon for help with flow cytometry, F. Chen and L. Kang for help with confocal imaging, N. Ji for assistance with C. elegans imaging, and B. Trout and C. Sudrik for help with spectroscopic analysis of iRFPs. We are grateful to X. Han and K. Hansen (Boston University) for the pCAGWPRE expression vector, and F. Subach (Moscow Institute of Physics and Technology) for the pWA23h plasmid. We are grateful to E. Costa, D. Estandian, and A. Wassie and L. Cai for useful discussions. C.S. acknowledges the Lefler Center for the Study of Neurodegenerative Disorders for support. E.S.B was supported by the HHMI-Simons Faculty Scholars Program, the IET Harvey Prize, the MIT Media Lab, the New York Stem Cell FoundationRobertson Award, the Open Philanthropy Project, Human Frontier Science Program RGP0015/2016, and NIH grants 1R43MH109332, 1R24MH106075, 2R01DA029639, 1R01EY023173, 1R01NS087950, 1R01MH103910, 1R01GM104948, and NIH Director's Pioneer Award 1DP1NS087724. O.S. was supported by a Simons Fellowship. H.-J.S. was supported by a Samsung Fellowship. D.G. was supported by an NSF Fellowship. Y.-G.Y. was supported by a Samsung Fellowship. L.F. was supported by a Simons Fellowship.

\section{References}

1. Packer MS, Liu DR. Methods for the directed evolution of proteins. Nat Rev Genet. 2015; 16:379394. [PubMed: 26055155]

2. Subach FV, Piatkevich KD, Verkhusha VV. Directed molecular evolution to design advanced red fluorescent proteins. Nat Methods. 2011; 8:1019-1026. [PubMed: 22127219]

3. Chen TW, et al. Ultrasensitive fluorescent proteins for imaging neuronal activity. Nature. 2013; 499:295-300. [PubMed: 23868258]

4. Klapoetke NC, et al. Independent optical excitation of distinct neural populations. Nat Methods. 2014; 11:338-46. [PubMed: 24509633]

5. Zhao Y, et al. Microfluidic cell sorter-aided directed evolution of a protein-based calcium ion indicator with an inverted fluorescent response. Integr Biol (Camb). 2014; 6:714-25. [PubMed: 24840546]

6. Dean KM, et al. Microfluidics-based selection of red-fluorescent proteins with decreased rates of photobleaching. Integr Biol. 2015; 7:263-73.

7. Fiedler BL, et al. Droplet Microfluidic Flow Cytometer for Sorting on Transient Cellular Responses of Genetically-Encoded Sensors. Anal Chem. 2017; 89:711-719. [PubMed: 27959493]

8. Lin MZ, Schnitzer MJ. Genetically encoded indicators of neuronal activity. Nat Neurosci. 2016; 19:1142-1153. [PubMed: 27571193]

9. Boyden ES. Optogenetics and the future of neuroscience. Nat Neurosci. 2015; 18:1200-1. [PubMed: 26308980]

10. Ai HW, Baird MA, Shen Y, Davidson MW, Campbell RE. Engineering and characterizing monomeric fluorescent proteins for live-cell imaging applications. Nat Protoc. 2014; 9:910-28. [PubMed: 24651502]

11. Chow BY, Chuong AS, Klapoetke NC, Boyden ES. Synthetic physiology: Strategies for adapting tools from nature for genetically targeted control of fast biological processes. Methods Enzymol. 2011; 497:425-443. [PubMed: 21601097]

12. Hochbaum DR, et al. All-optical electrophysiology in mammalian neurons using engineered microbial rhodopsins. Nat Methods. 2014; 11:825-33. [PubMed: 24952910] 
13. Flytzanis NC, et al. Archaerhodopsin variants with enhanced voltage-sensitive fluorescence in mammalian and Caenorhabditis elegans neurons. Nat Commun. 2014; 5:4894. [PubMed: 25222271]

14. St-Pierre F, et al. High-fidelity optical reporting of neuronal electrical activity with an ultrafast fluorescent voltage sensor. Nat Neurosci. 2014; 17:884-9. [PubMed: 24755780]

15. Wagner MJ, Li JZ, Gong Y, Schnitzer MJ. FRET-opsin protein voltage sensors. Nat Commun. 2014; 5:1-11.

16. Gong Y, et al. High-speed recording of neural spikes in awake mice and flies with a fluorescent voltage sensor. Sciencexpress. 2015; 350:1-11.

17. Környei Z, et al. Cell sorting in a Petri dish controlled by computer vision. Sci Rep. 2013; 3:1088. [PubMed: 23336070]

18. Salánki R, et al. Automated single cell sorting and deposition in submicroliter drops. Appl Phys Lett. 2014; 105:83703.

19. Giraud E, et al. Bacteriophytochrome controls photosystem synthesis in anoxygenic bacteria. Nature. 2002; 417:202-205. [PubMed: 12000965]

20. He L, Friedman AM, Bailey-Kellogg C. A divide-and-conquer approach to determine the Pareto frontier for optimization of protein engineering experiments. Proteins Struct Funct Bioinforma. 2012; 80:790-806.

21. Knowles JD, Corne DW. Approximating the nondominated front using the Pareto Archived Evolution Strategy. Evol Comput. 2000; 8:149-172. [PubMed: 10843519]

22. Currin A, Swainston N, Day PJ, Kell DB. Synthetic biology for the directed evolution of protein biocatalysts: navigating sequence space intelligently. Chem Soc Rev. 2015; 44:1172-239. [PubMed: 25503938]

23. Grigoryan G, Reinke AW, Keating AE. Design of protein-interaction specificity gives selective bZIP-binding peptides. Nature. 2009; 458:859-864. [PubMed: 19370028]

24. McIsaac RS, et al. Directed evolution of a far-red fluorescent rhodopsin. Proc Natl Acad Sci. 2014; 105:6374-9.

25. Kotnik T, Pucihar G, Miklavčič D. Induced transmembrane voltage and its correlation with electroporation- mediated molecular transport. J Membr Biol. 2010; 236:3-13. [PubMed: 20617432]

26. Del Re AM, Woodward JJ. Inhibition of gap junction currents by the abused solvent toluene. Drug Alcohol Depend. 2005; 78:221-224. [PubMed: 15845326]

27. Gradinaru V, et al. Molecular and Cellular Approaches for Diversifying and Extending Optogenetics. Cell. 2010; 141:154-165. [PubMed: 20303157]

28. Chuong AS, et al. Noninvasive optical inhibition with a red-shifted microbial rhodopsin. Nat Neurosci. 2014; 17:1123-9. [PubMed: 24997763]

29. Shemesh O, et al. Temporally precise single-cell resolution optogenetics. Nat Neurosci. 2017; 20:1796-1806. [PubMed: 29184208]

30. Friedrich RW, Jacobson GA, Zhu P. Circuit Neuroscience in Zebrafish. Curr Biol. 2010; 20:371381.

31. Stewart AM, Braubach O, Spitsbergen J, Gerlai R, Kalueff AV. Zebrafish models for translational neuroscience research: From tank to bedside. Trends Neurosci. 2014; 37:264-278. [PubMed: 24726051]

32. Ahrens MB, et al. Brain-wide neuronal dynamics during motor adaptation in zebrafish. Nature. 2012; 485:471-477. [PubMed: 22622571]

33. Ahrens MB, Orger MB, Robson DN, Li JM, Keller PJ. Whole-brain functional imaging at cellular resolution using light-sheet microscopy. Nat Methods. 2013; 10:413-20. [PubMed: 23524393]

34. Wyart C, et al. Optogenetic dissection of a behavioural module in the vertebrate spinal cord. Nature. 2009; 461:407-410. [PubMed: 19759620]

35. Gordus A, et al. Feedback from Network States Generates Variability in a Probabilistic Olfactory Circuit. Cell. 2015; 161:215-227. [PubMed: 25772698]

36. Dobes NC, et al. Laser-based directed release of array elements for efficient collection into targeted microwells. Analyst. 2013; 138:831-838. [PubMed: 23223411] 
37. Dean KM, et al. Microfluidics-based selection of red-fluorescent proteins with decreased rates of photobleaching. Integr Biol. 2015; 7:263-73.

38. Bouchard MB, et al. Swept confocally-aligned planar excitation (SCAPE) microscopy for high speed volumetric imaging of behaving organisms. Nat Photonics. 2015; 9:113-119. [PubMed: 25663846]

39. Prevedel R, et al. Simultaneous whole-animal 3D imaging of neuronal activity using light-field microscopy. Nat Methods. 2014; 11:727-30. [PubMed: 24836920]

40. Krzywinski M, Altman N. Points of Significance: Visualizing samples with box plots. Nat Methods. 2014; 11:119-120. [PubMed: 24645192]

41. Subedi A, et al. Adoption of the $\mathrm{Q}$ transcriptional regulatory system for zebrafish transgenesis. Methods. 2014; 66:433-440. [PubMed: 23792917]

42. Piatkevich KD, Subach FV, Verkhusha VV. Far-red light photoactivatable near-infrared fluorescent proteins engineered from a bacterial phytochrome. Nat Commun. 2013; 4:2153. [PubMed: 23842578]

43. Filonov GS, et al. Bright and stable near-infrared fluorescent protein for in vivo imaging. Nat Biotechnol. 2011; 29:757-761. [PubMed: 21765402]

44. Makarov NS, Drobizhev M, Rebane A. Two-photon absorption standards in the 550-1600 nm excitation wavelength range. Opt Express. 2008; 16:4029-4047. [PubMed: 18542501]

45. Drobizhev M, Makarov NS, Tillo SE, Hughes TE, Rebane A. Two-photon absorption properties of fluorescent proteins. Nat Methods. 2011; 8:393-399. [PubMed: 21527931]

46. Lebkowski JS, DuBridge RB, Antell Ea, Greisen KS, Calos MP. Transfected DNA is mutated in monkey, mouse, and human cells. Mol Cell Biol. 1984; 4:1951-1960. [PubMed: 6095032]

47. Mahon MJ. Vectors bicistronically linking a gene of interest to the SV40 large T antigen in combination with the SV40 origin of replication enhance transient protein expression and luciferase reporter activity. Biotechniques. 2011; 51:119-126. [PubMed: 21806556]

48. Qin JY, et al. Systematic comparison of constitutive promoters and the doxycycline-inducible promoter. PLoS One. 2010; 5:3-6.

49. Chen C, Okayama H. High-efficiency transformation of mammalian cells by plasmid DNA. Mol Cell Biol. 1987; 7:2745-2752. [PubMed: 3670292]

50. Okazaki M, Yoshida Y, Yamaguchi S, Kaneno M, Elliott JC. Affinity binding phenomena of DNA onto apatite crystals. Biomaterials. 2001; 22:2459-2464. [PubMed: 11516076]

51. Pucihar G, Kotnik T, Miklavcic D. Measuring the induced membrane voltage with Di-8-ANEPPS. J Vis Exp. 2009; 88:4-6.

52. Chow BY, et al. High-performance genetically targetable optical neural silencing by light-driven proton pumps. Nature. 2010; 463:98-102. [PubMed: 20054397]

53. Jiang M, Chen G. High Ca2+-phosphate transfection efficiency in low-density neuronal cultures. Nat Protoc. 2006; 1:695-700. [PubMed: 17406298]

54. Kimura Y, Satou C, Higashijima SI. V2a and V2b neurons are generated by the final divisions of pair-producing progenitors in the zebrafish spinal cord. Development. 2008; 135:3001-3005. [PubMed: 18684740]

55. Kwan KM, et al. The Tol2kit: A multisite gateway-based construction Kit for Tol2 transposon transgenesis constructs. Dev Dyn. 2007; 236:3088-3099. [PubMed: 17937395]

56. Fisher $\mathrm{S}$, et al. Evaluating the biological relevance of putative enhancers using Tol 2 transposonmediated transgenesis in zebrafish. Nat Protoc. 2006; 1:1297-1305. [PubMed: 17406414]

57. Renaud O, Herbomel P, Kissa K. Studying cell behavior in whole zebrafish embryos by confocal live imaging: application to hematopoietic stem cells. Nat Protoc. 2011; 6:1897-904. [PubMed: 22082984]

58. Brenner S. The genetics of Caenorhabditis elegans. Genetics. 1974; 77:71-94. [PubMed: 4366476]

59. Kralj JM, Douglass AD, Hochbaum DR, Maclaurin D, Cohen AE. Optical recording of action potentials in mammalian neurons using a microbial rhodopsin. Nat Methods. 2012; 9:90-95.

60. Lee DD, Seung HS. Learning the parts of objects by non-negative matrix factorization. Nature. 1999; 401:788-91. [PubMed: 10548103] 
61. Dell RB, Holleran S, Ramakrishnan R. Sample size determination. ILAR J. 2002; 43:207-13. [PubMed: 12391396] 
a
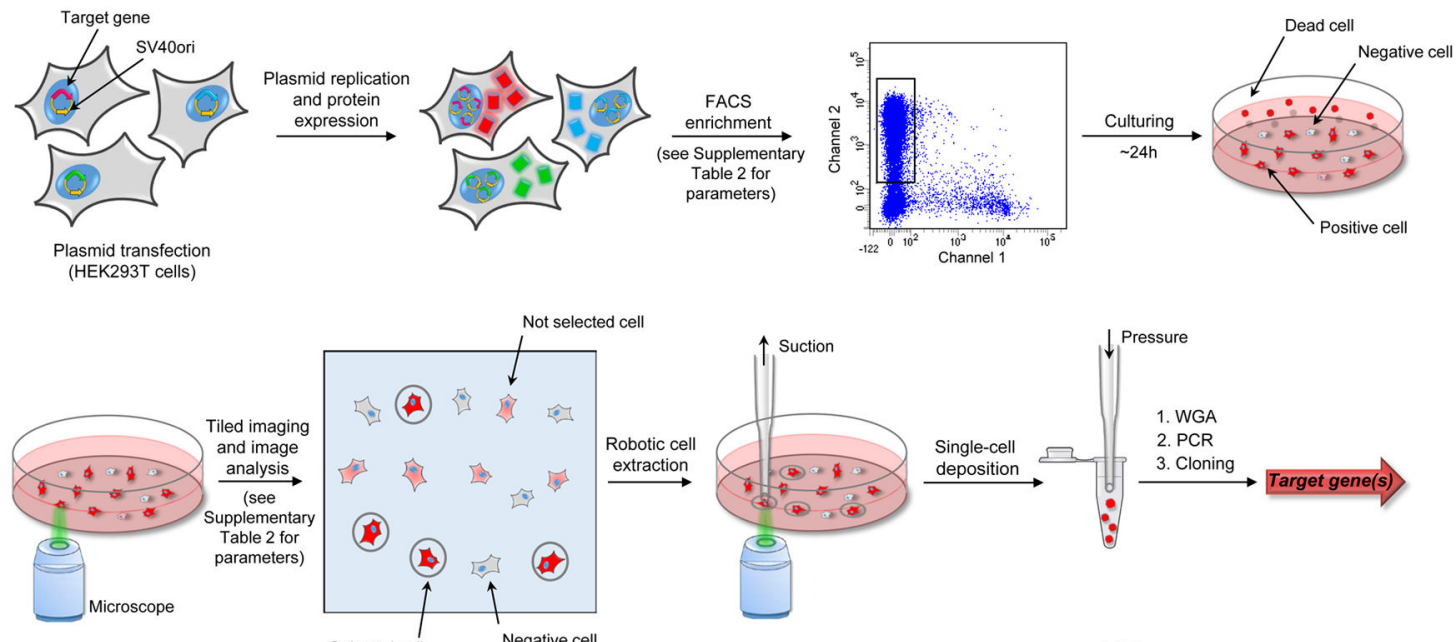

b
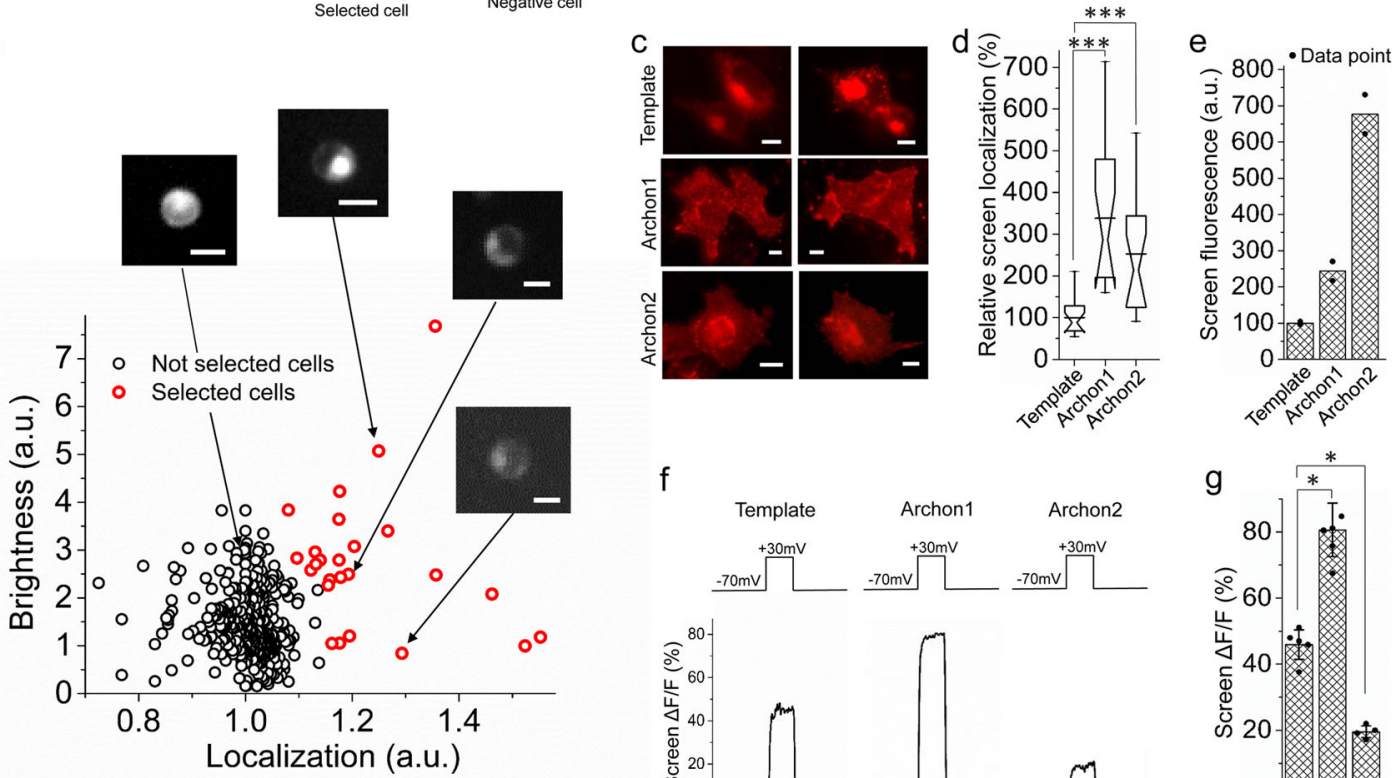

f
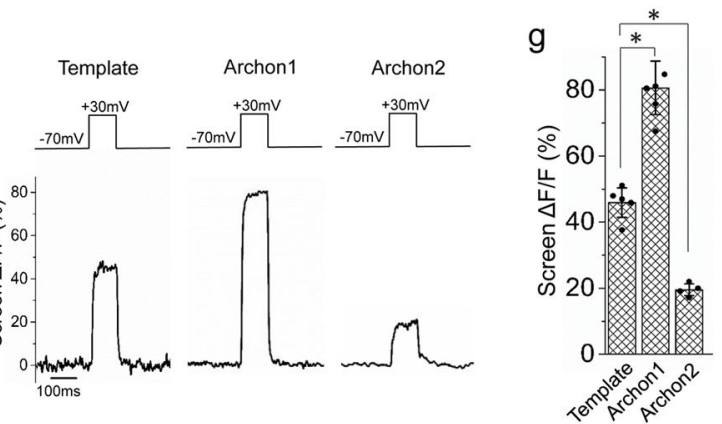

Figure 1. Multi-parameter directed evolution of proteins in mammalian cells via robotic cell picking

(a) Pipeline for multi-parameter directed evolution of proteins in mammalian cells using robotic cell picking. Abbreviations: FACS, fluorescence-activating cell sorting (FACS);

WGA, whole-genome amplification. (b) Example data and analyses reflecting the quantitative metrics used in the cell-picker step during the second round of directed evolution, for simultaneous optimization of brightness and localization. Scale bar: $10 \mu \mathrm{m}$. (c) Representative fluorescence images of HEK293T cells expressing the template, Archon1 and Archon2 ( $\mathrm{n}=15,16$, and 16 cells for Archon1, Archon2, and the template respectively). Dynamic ranges for the images were normalized to facilitate visual comparison. Scale bars, $5 \mu \mathrm{m}$. Imaging conditions: $62 \mathrm{~mW} / \mathrm{mm}^{2}, \lambda_{\mathrm{ex}}=628 / 31 \mathrm{BP}$ (bandpass, used throughout) from an LED, $\lambda_{\mathrm{em}}=664 \mathrm{LP}$ (longpass, used throughout) used in (c,d). (d) Relative membrane localization of the indicators of $\mathbf{c}$ in HEK293T cells $(n=15,16$, and 16 cells for Archon1, 
Archon2, and the template respectively, each from 2 independent transfections; $* * * P<$ 0.0001 for Archon 1 and $* * * P=0.0003$ for Archon2, Kruskal-Wallis analysis of variance followed by post-hoc test via Steel's test with the template as control group). Box plots with notches are used throughout this paper, when $\mathrm{n}>6$, as recommended by ref ${ }^{40}$ (narrow part of notch, median; top and bottom of the notch, $95 \%$ confidence interval for the median; top and bottom horizontal lines, $25 \%$ and $75 \%$ percentiles for the data; whiskers extend 1.5 times the interquartile range from the 25 th and 75 th percentiles; horizontal line, mean). (e) FACS mean fluorescence intensity for sets of live HEK293T cells expressing these indicators ( $\mathrm{n}=2$ transfected samples, each; individual data points in black dots). (f) Representative fluorescence changes for these indicators with a $100 \mathrm{mV}$ voltage step, measured in HEK293T cells. Imaging conditions: $\lambda_{\mathrm{ex}}=637 \mathrm{~nm}$ laser light, $\lambda_{\mathrm{em}}=664 \mathrm{LP}, 800$ $\mathrm{mW} / \mathrm{mm}^{2}$ used for the template and $80-800 \mathrm{~mW} / \mathrm{mm}^{2}$ used for Archons in (f,g), with light intensity adjusted to prevent signal saturation. (g) Population data of fluorescence changes, as in $\mathbf{f}$, for these indicators $(n=5,6$, and 4 cells for the template, Archon1, and Archon2, each from 2 independent transfections; individual data points in black dots; error bars, standard deviation; $* P=0.0155$ for Archon 1 and $* P=0.0374$ for Archon2, Kruskal-Wallis analysis of variance followed by post-hoc Steel's test with the template as control group), taken in the steady state. 

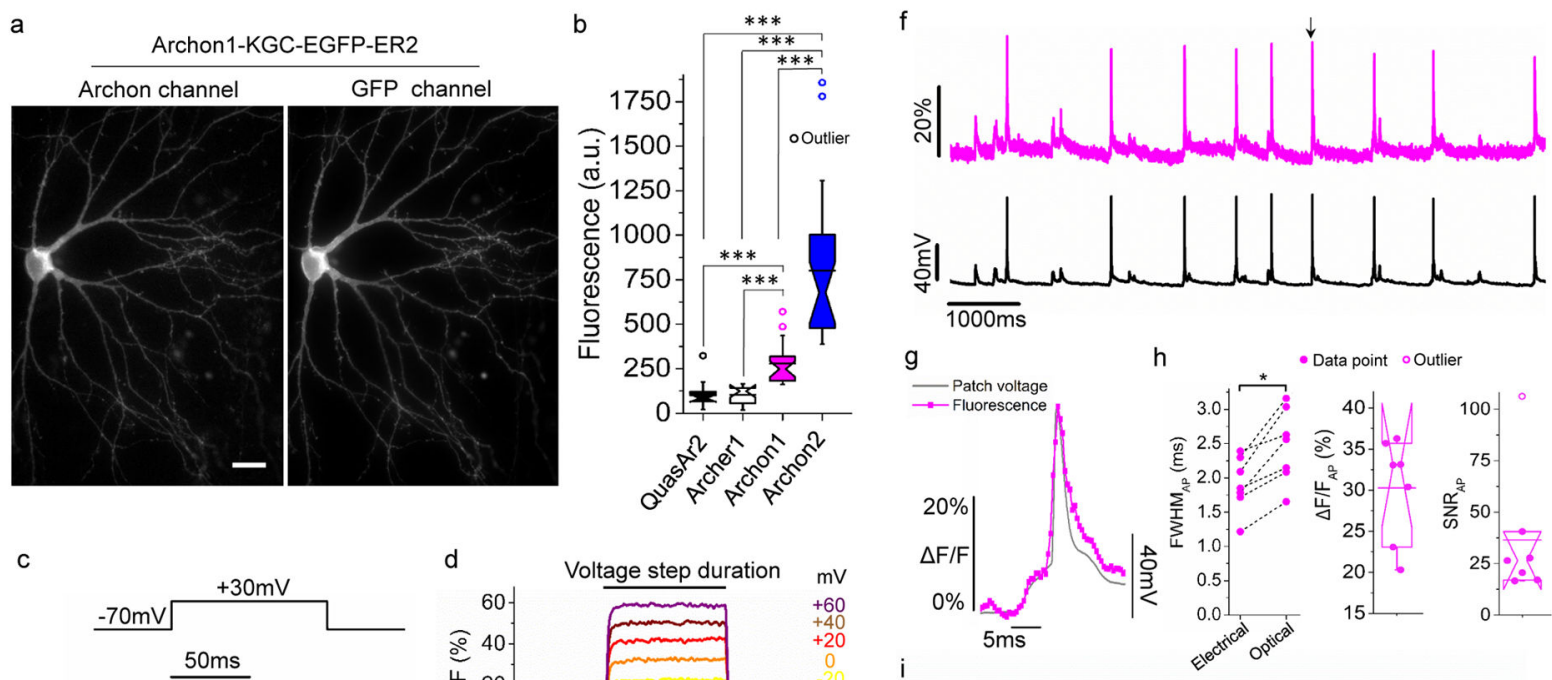
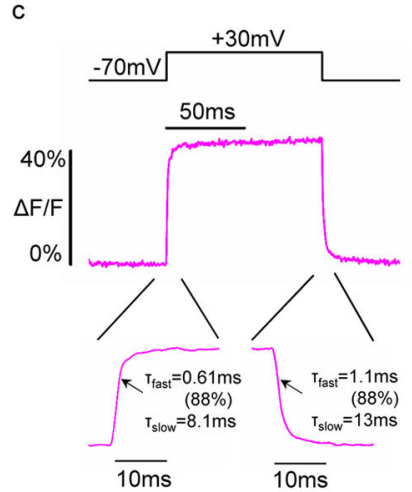

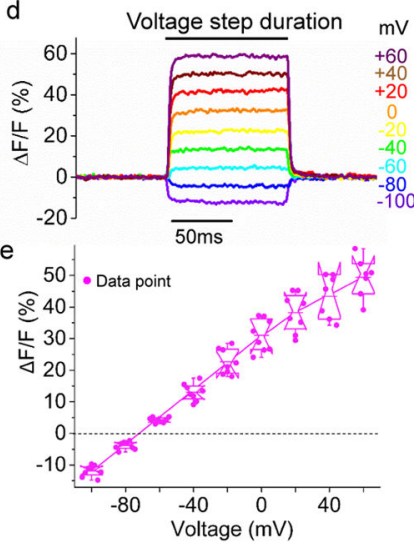

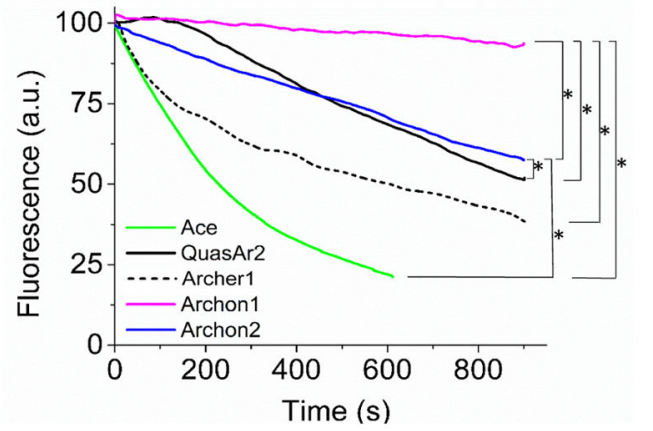

Figure 2. Characterization of Archons in cultured cells

(a) Representative fluorescent images of Archon1 (left, excitation $\left(\lambda_{\mathrm{ex}}\right)$ with $637 \mathrm{~nm}$ laser light, emission $\left(\lambda_{\mathrm{em}}\right)$ at 664LP) and GFP (right, $\lambda_{\mathrm{ex}}=475 / 34 \mathrm{BP}$ from an LED and $\lambda_{\mathrm{em}}=$ $527 / 50 \mathrm{BP})$ channels in a cultured mouse hippocampal neuron $(\mathrm{n}=32$ cells from 5 independent transfections). Scale bar: $10 \mu \mathrm{m}$. (b) Relative fluorescence of QuasAr2, Archer1, Archon1, and Archon2 in cultured neurons $(\mathrm{n}=18,16,23$, and 23 cells respectively, from 4 independent transfections each, from one culture; $\lambda_{\mathrm{ex}}=637 \mathrm{~nm}$ laser light at $800 \mathrm{~mW} / \mathrm{mm}^{2}$ and $\lambda_{\mathrm{em}}=664 \mathrm{LP}$ for Fig. $2 \mathrm{c}-\mathrm{g}$; $* * * P<0.0001$, Kruskal-Wallis analysis of variance followed by post-hoc Steel-Dwass test on each pair; see Supplementary Table 5 for full statistics for Fig. 2). Box plots with notches are used (see caption of Fig. 1d for description). Open circles represent outliers, data points which are less than $25^{\text {th }}$ percentile or greater than $75^{\text {th }}$ percentile by more than 1.5 times the interquartile range. (c) Representative fluorescence response of Archon 1 in a cultured neuron, to a $100 \mathrm{mV}$ change delivered in voltage-clamp. $\tau_{\text {fast }}$ and $\tau_{\text {slow }}$ indicate time constants with the fluorescence trace

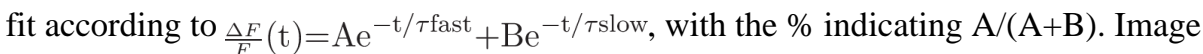
acquisition rate: $3.2 \mathrm{kHz}$. (d) Representative fluorescence traces of Archon1 in response to a series of voltage steps in voltage-clamp mode. Image acquisition rate: $2.3 \mathrm{kHz}$. (e) Population data corresponding to the experiment of $\mathbf{d}$ ( $\mathrm{n}=8$ neurons from 3 cultures). Data was normalized so that $-70 \mathrm{mV}$ was set to $0 \Delta \mathrm{F} / \mathrm{F}$. (f) Single-trial optical recording of Archon1 fluorescence responses (magenta) during spontaneous activity, with concurrent current clamp trace (black), for a cultured hippocampal neuron. Peak marked with arrow is 
zoomed-in in (g). Image acquisition rate: $2.3 \mathrm{kHz}$. (g) Zoomed-in view of peak marked with arrow in (f), scaled to match peaks. (h) Quantification of electrical and optical full width at half maximum (FWHM; dashed lines connect data points from same neuron), $\Delta \mathrm{F} / \mathrm{F}$, and signal-to-noise ratio (SNR), per action potential (AP) across all recordings ( $\mathrm{n}=160$ APs from 7 neurons from 5 cultures). $* P=0.0156$, Wilcoxon signed-rank test. (i) Photobleaching curves of Ace, QuasAr2, Archer1, Archon1 and Archon2 under continuous illumination ( $\mathrm{n}=$ 5, 7, 5, 9, and 7 neurons from 1, 1, 1, 2, and 2 cultures, respectively; 475/34BP from an LED at $13 \mathrm{~mW} / \mathrm{mm}^{2}$ for Ace $2 \mathrm{~N}-4$ aa-mNeon, $637 \mathrm{~nm}$ laser light at $2.2 \mathrm{~W} / \mathrm{mm}^{2}$ for QuasAr2 and Archer1, $637 \mathrm{~nm}$ laser light at $800 \mathrm{~mW} / \mathrm{mm}^{2}$ for Archon1 and Archon2; light intensity was adjusted to have the same initial signal-to-noise ratio (SNR) of action potentials, e.g. 25 \pm 8 , $26 \pm 12,26 \pm 10,26 \pm 10$ and $28 \pm 7$ for Quasar2, Archer1, Archon1, Archon2 and Ace2N-4aamNeon respectively; image acquisition rate: $333 \mathrm{~Hz}$ ); ${ }^{*} P=0.0184$ for Archon 1 and Archon2, Archon1 and QuasAr2, and Archon2 and QuasAr2; * $P=0.0456$ for Archon1 and Archer1, Archon 1 and Ace, and Archon2 and Ace; Kruskal-Wallis analysis of variance of bleaching time followed by post-hoc Steel-Dwass test on each pair). 
a
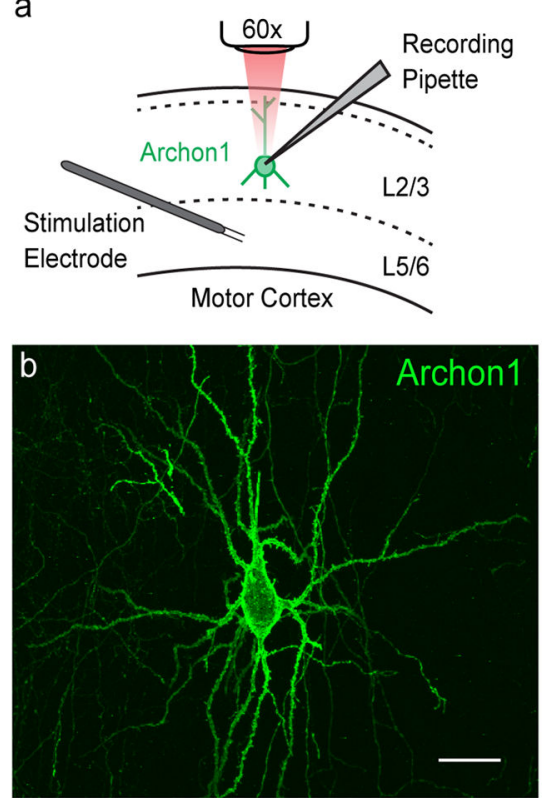

g

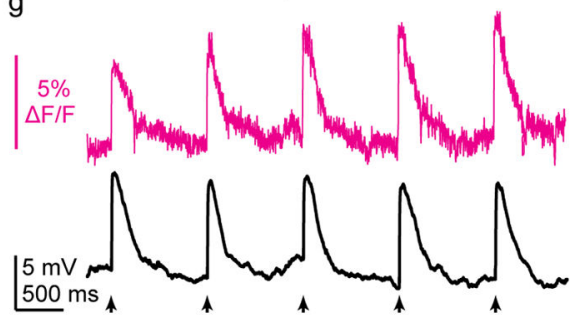

c

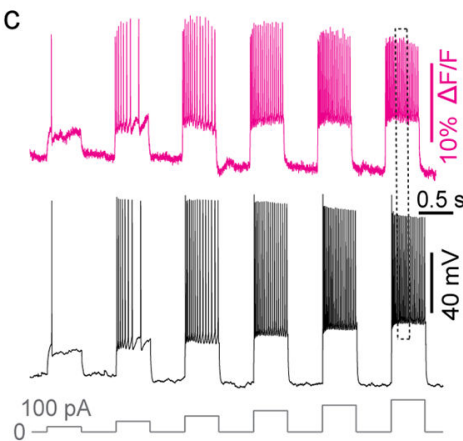

d

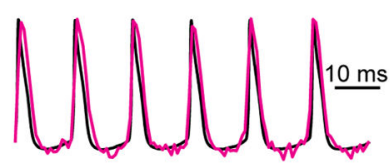

e

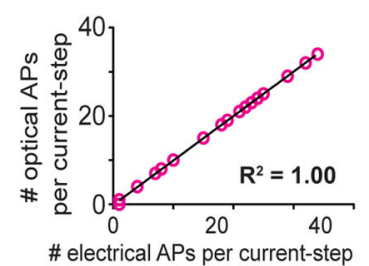

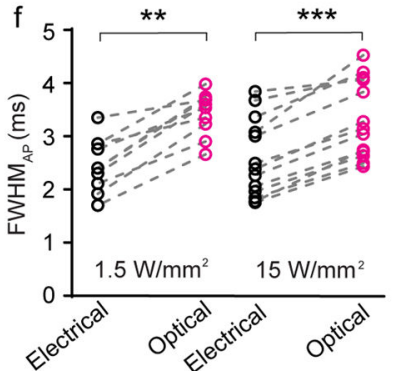

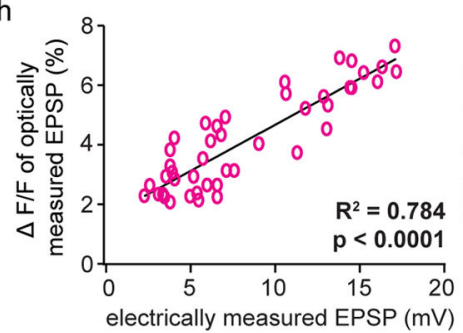

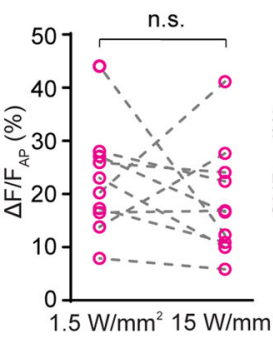
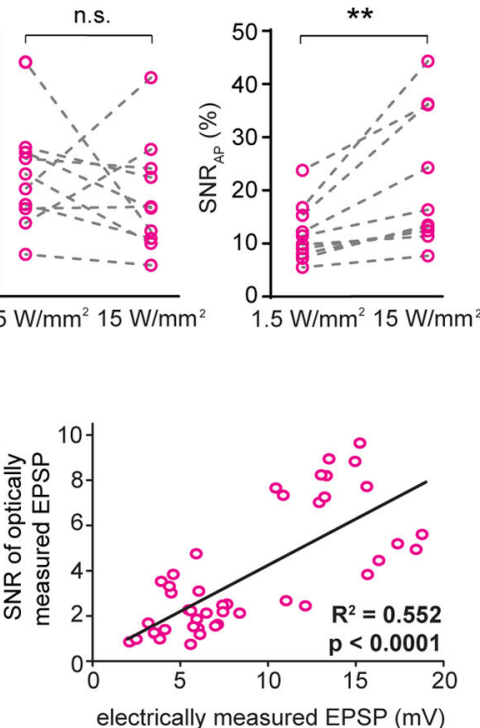

Figure 3. Millivolt-scale imaging of neural voltage in intact brain slices

(a) Schematic of experimental recording configuration. Archon1-expressing pyramidal neurons in layer (L) 2/3 of motor cortex were targeted by patch-clamp recording, and Archon fluorescence at the soma was imaged at $1 \mathrm{kHz}$. Excitation intensity was $\sim 7 \mathrm{~mW}$ over the area of the soma (i.e., $\sim 15 \mathrm{~W} / \mathrm{mm}^{2}$ at $637 \mathrm{~nm}$, but 10x lower intensity, $1.5 \mathrm{~W} / \mathrm{mm}^{2}$ at $637 \mathrm{~nm}$, was used in Fig. 3f for comparison). A bipolar stimulation electrode was in some experiments placed in L5 to trigger excitatory synaptic events in Archon1-expressing L2/3 pyramidal neurons. (b) Representative image of Archon1 expressing neuron in L2/3 of mouse motor cortex $(n=70$ slices from 3 mice). Scale bar: $25 \mu \mathrm{m}$. (c) A series of $500 \mathrm{~ms}$ current steps with increasing amplitudes (from 100 to 600 pA, in 100pA increments; gray line) were injected through the recording pipette, resulting in action potentials of varying frequency. Magenta, imaged trace; black, simultaneous whole-cell patch-clamp in current clamp mode. (d) Zoomed-in view of APs marked with dotted box in c, scaled to match peaks. (e) Graph of the number of optically detected APs vs. the number of electrically detected APs for every 500 ms-long current injection across all cells that underwent the experiment of $\mathbf{c}(\mathrm{n}=22$ steps from 5 neurons); straight line indicates linear regression. (f) Quantification of electrical and optical full width at half maximum (FWHM, left), $\Delta \mathrm{F} / \mathrm{F}$ (middle), and SNR (right) for action potentials; $\mathrm{n}=10$ neurons from 6 mice; means are plotted for each cell; dashed lines connect data points from same neuron; Wilcoxon signed- 
rank test: $* * P=0.002$ and $* * * P=0.0002$ for FWHM, $P=0.375$ (not significant, n.s.) for $\Delta \mathrm{F} / \mathrm{F}, * * P=0.002$ for SNR, see Supplementary Table 5 for full statistics for Fig. 3. (g) Representative optical (magenta) and electrical (black) signals from electrically evoked excitatory postsynaptic potentials (EPSPs). Arrows indicate times of stimulation ( 5 stimuli at $1 \mathrm{~Hz}$, followed by inter-trial intervals of $>30$ seconds). (h) Population data of $\Delta \mathrm{F} / \mathrm{F}$ (left) and SNR (right) from individual EPSPs as in (g) across all cells ( $\mathrm{n}=45$ EPSPs from 4 neurons from 2 mice); straight line indicates linear regression. 

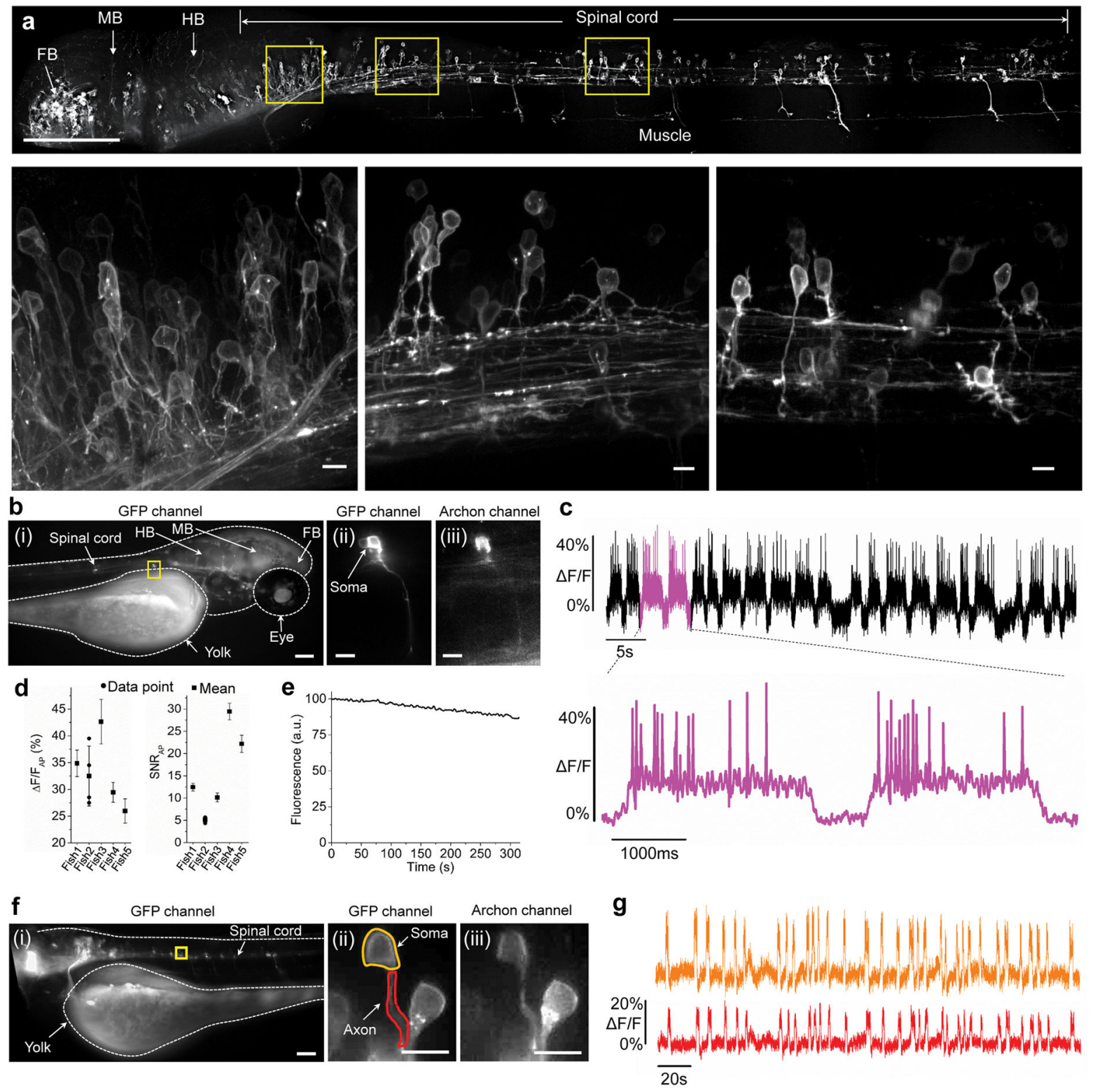

Figure 4. Voltage imaging of Archon1-expressing neurons in larval zebrafish

a Representative fluorescence image (top; GFP channel: excitation $\left(\lambda_{\mathrm{ex}}\right)$ at $465 \mathrm{~nm}$ laser light, emission ( $\left.\lambda_{\mathrm{em}}\right)$ at $527 / 50 \mathrm{BP}$ ) of neurons expressing zArchon1-EGFP in the spinal cord of a zebrafish larva at 3 days post fertilization (dpf) $(n=4$ fish). Yellow boxes indicate neurons zoomed-in in the bottom panels. Scale bar, $125 \mu \mathrm{m}$. FB, forebrain; MB, midbrain; HB, hindbrain. (Bottom) From left to right: high magnification images of the neurons highlighted in the yellow boxes in the top panel. Scale bar, $5 \mu \mathrm{m}$. (b) Representative image (i, $\lambda_{\mathrm{ex}}=475 / 34 \mathrm{BP}$ from an LED, $\lambda_{\mathrm{em}}=527 / 50 \mathrm{BP}$ ) of neurons expressing zArchon1-GFP in the spinal cord of a $4 \mathrm{dpf}$ zebrafish larva immobilized in agarose under wide-field microscopy ( $\mathrm{n}=5$ fish). The yellow box indicates a neuron zoomed in in later panels. Scale bar: $100 \mu \mathrm{m}$. (ii) High magnification image of the neuron highlighted in the yellow box of (i) in the GFP channel. Scale bar: $10 \mu \mathrm{m}$. (iii) As in ii, but in the Archon $\left(\lambda_{\mathrm{ex}}=637 \mathrm{~nm}\right.$ laser 
light, $\lambda_{\mathrm{em}}=664 \mathrm{LP}$ ) channel. (c) Representative fluorescence trace (top) of zArchon1 reporting spontaneous activity of the neuron shown in $\mathbf{b}$ (at soma; $\lambda_{\mathrm{ex}}=637 \mathrm{~nm}$ laser light at $2.2 \mathrm{~W} / \mathrm{mm}^{2}, \lambda_{\mathrm{em}}=664 \mathrm{LP}$; image acquisition rate: $500 \mathrm{~Hz} ; \mathrm{n}=5$ neurons from $5 \mathrm{fish}$ ).

Bottom, the section of $\mathbf{b}$ highlighted in magenta, shown at an expanded time scale. (d) Population data of fluorescence changes and signal-to-noise ratios of zArchon1 during action potentials (APs; $\mathrm{n}=21,4,132,71$ and 58 action potentials for fish 1-5, respectively; plotted is mean and standard deviation for each fish; raw data points are shown for fish with $\mathrm{n}<10$ APs). (e) Photobleaching of zArchon 1 fluorescence measured in in vivo in zebrafish larvae ( $\mathrm{n}=11$ neurons from 6 fish) over $300 \mathrm{~s}$ of continuous illumination at $2.2 \mathrm{~W} / \mathrm{mm}^{2}$. (f) Representative image (in the GFP channel) of neurons expressing zArchon1 in the spinal cord of a zebrafish larva at $4 \mathrm{dpf}$ immobilized in agarose under wide-field microscopy ( $\mathrm{n}=3$ fish). A yellow box indicates neurons zoomed in in later panels. Scale bar: 100 $\mu$ m. (ii) High magnification image of the neurons highlighted in the yellow box of (i) in the GFP channel. Scale bar: 10um. Highlighted regions indicate the soma (yellow) and the axon (red) of the neuron of interest. (iii) As in ii, but in the Archon channel. (g) Representative fluorescence trace of zArchon1 reporting spontaneous activity at the soma and the axon of the neuron shown in $\mathbf{f}$ ( $\mathrm{n}=3$ neurons from 3 fish). The traces were acquired at the soma (yellow) and the axon (red) of the neuron $\left(\lambda_{\mathrm{ex}}=637 \mathrm{~nm}\right.$ laser light at $2.2 \mathrm{~W} / \mathrm{mm}^{2}, \lambda_{\mathrm{em}}=664 / \mathrm{LP}$, image acquisition rate: $250 \mathrm{~Hz}$ ). 
a
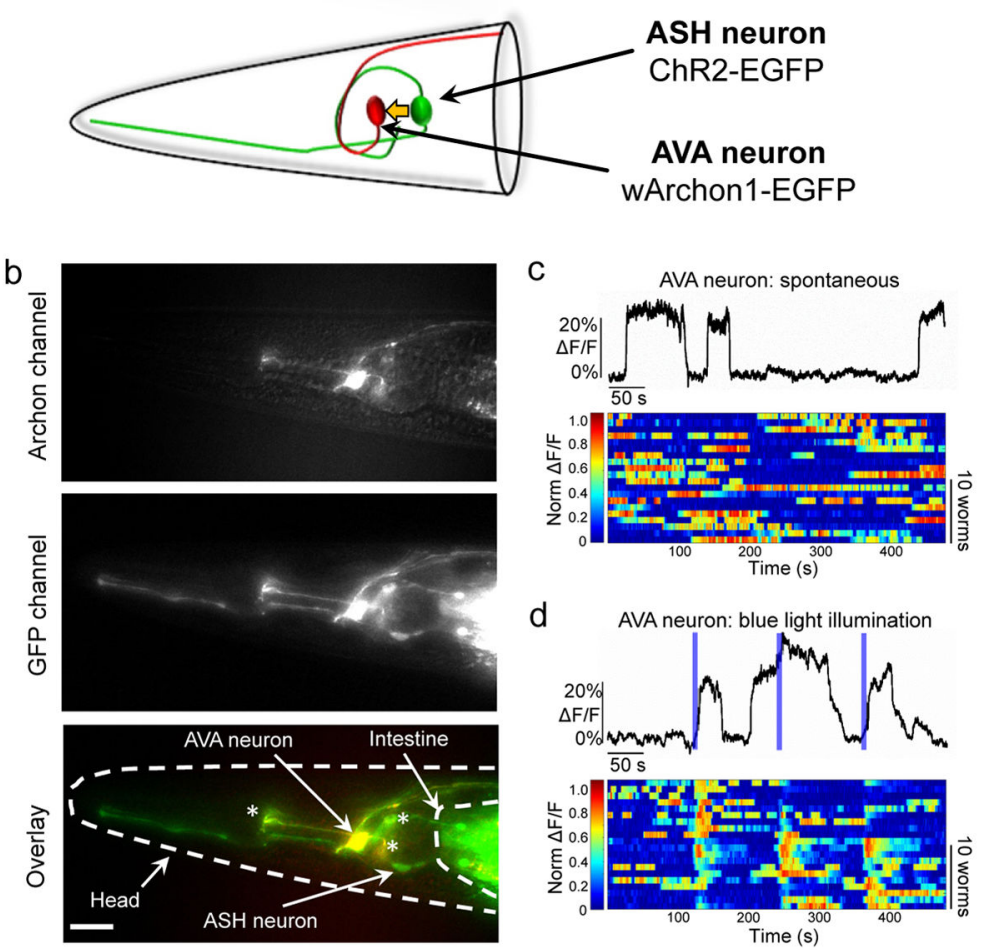

e

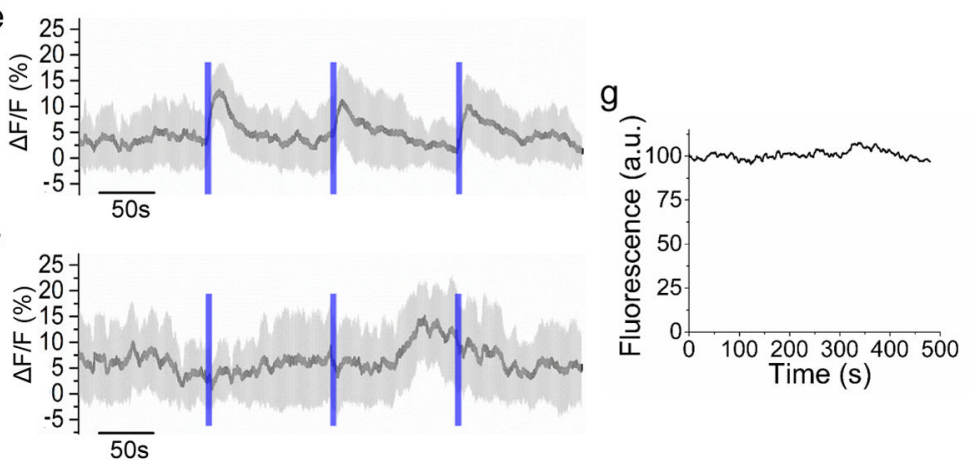

Figure 5. All-optical electrophysiology in C. elegans

(a) Schematic of AVA neuron expressing wArchon1-EGFP (red) and ASH neuron expressing ChR2-EGFP (green), in the head of $C$. elegans. A yellow arrow indicates synaptic connection from ASH onto AVA. (b) Fluorescence images of the $C$. elegans head expressing wArchon1-EGFP in an AVA neuron (under rig-3 promoter) and ChR2-GFP (under sra-6 promoter) in the ASH neuron (top, Archon channel; middle, GFP channel; bottom, overlay), as well as pharyngeal neurons that express wArchon1-EGFP under control of the rig-3 promoter (asterisks; $\mathrm{n}=20$ worms). Scale bar: $20 \mu \mathrm{m}$. (c; top) A representative trace of wArchon1 fluorescence reporting spontaneous activity in the soma of an AVA neuron ( $\mathrm{n}=20$ cells from 20 worms). (Bottom) Individual traces of wArchon1 fluorescence reporting spontaneous activity in an AVA neuron ( $\mathrm{n}=20$ neurons in 20 worms). (d; top) A representative trace of wArchon 1 fluorescence in soma of an AVA neuron under three pulses of blue light stimulation $\left(0.2 \mathrm{~mW} / \mathrm{mm}^{2}, \lambda_{\mathrm{ex}}=475 / 34 \mathrm{BP}\right.$ light from an LED, $6 \mathrm{~s}$; blue bars $)$. 
(Bottom) Individual traces of wArchon1 fluorescence in an AVA neuron under blue light illumination ( $\mathrm{n}=20$ neurons in 20 worms). (e) Averaged wArchon1 fluorescence changes for traces presented in panel d. Shaded area is standard deviation. (f) Averaged wArchon1 fluorescence changes for traces recorded under same conditions as in panel $\mathrm{d}$ using the worms expressing only wArchon1-EGFP in AVA neurons. Shaded area is standard deviation. (g) Photobleaching curve of wArchon1 expressed in AVA neurons under continuous $637 \mathrm{~nm}$ excitation illumination ( $\mathrm{n}=10$ cells from 10 worms, $\lambda_{\mathrm{ex}}=637 \mathrm{~nm}$ laser light at $\left.800 \mathrm{~mW} / \mathrm{mm}^{2}, \lambda_{\mathrm{em}}=664 \mathrm{LP}\right)$. 